Math. Model. Nat. Phenom.

Vol. 3, No. 7, 2008, pp. 194-228

\title{
The Basic Reproduction Number of an Infectious Disease in a Stable Population: The Impact of Population Growth Rate on the Eradication Threshold
}

\author{
H. Inaba ${ }^{a 1}$ and H. Nishiura ${ }^{b}$ \\ ${ }^{a}$ Graduate School of Mathematical Sciences, University of Tokyo, \\ 3-8-1 Komaba, Meguro-ku, Tokyo 153-8914, Japan \\ $b$ Theoretical Epidemiology, Faculty of Veterinary Medicine, University of Utrecht, \\ Yalelaan 7, 3584 CL, Utrecht, The Netherlands
}

\begin{abstract}
Although age-related heterogeneity of infection has been addressed in various epidemic models assuming a demographically stationary population, only a few studies have explicitly dealt with age-specific patterns of transmission in growing or decreasing population. To discuss the threshold principle realistically, the present study investigates an age-durationstructured SIR epidemic model assuming a stable host population, as the first scheme to account for the non-stationality of the host population. The basic reproduction number $R_{0}$ is derived using the next generation operator, permitting discussions over the well-known invasion principles. The condition of endemic steady state is also characterized by using the effective next generation operator. Subsequently, estimators of $R_{0}$ are offered which can explicitly account for non-zero population growth rate. Critical coverages of vaccination are also shown, highlighting the threshold condition for a population with varying size. When quantifying $R_{0}$ using the force of infection estimated from serological data, it should be remembered that the estimate increases as the population growth rate decreases. On the contrary, given the same $R_{0}$, critical coverage of vaccination in a growing population would be higher than that of decreasing population. Our exercise implies that high mass vaccination coverage at an early age would be needed to control childhood vaccine-preventable diseases in developing countries.
\end{abstract}

Key words: epidemiological model, stable age distribution, threshold, basic reproduction number, SIR model

AMS subject classification: 92D25, 92D30

\footnotetext{
${ }^{1}$ Corresponding author. Email: inaba@ms.u-tokyo.ac.jp
} 


\section{Introduction}

To discuss potential control strategies of an infectious disease, the basic reproduction number, $R_{0}$, has become a central quantity to determine the intensity of interventions $[12] . R_{0}$ has been effectively applied to epidemiological practice in relation to vaccination, yielding threshold coverage of vaccination to eradicate a disease [1]. To appropriately understand the threshold condition, we have learnt that it is essential to capture realistic patterns of infectious disease spread, addressing various heterogeneities of transmission [11], and especially, age-related heterogeneity has been one of the most important topics which were theoretically explored in detail [26]. Given the theoretical basis, various practical and quantitative methods have been proposed, which for example use age-specific seroprevalence data [17], permitting statistical estimation of $R_{0}$, and addressing age-related heterogeneity by conveniently assuming proportionate mixing [14] or by constructing "who acquires infection from whom (WAIFW)" matrix in discrete age-groups [15]. Applying these techniques to the observed data, vaccination strategies against various childhood viral diseases have been discussed previously ([16], [22], [46]). Statistical methods have been extended in the relevant issue, widening the practical applicability to estimate $R_{0}$ in public health practice. For example, contact surface model in continuous age scale is likely to yield precise estimate of the force of infection [18], and with regard to the inference, a recent study proposed a method to use social contact data to estimate the age-specific transmission parameters [54].

Nevertheless, the majority of previous studies addressing age-related heterogeneity tended to assume a demographically stationary host population, and there are only a few models which highlighted non-stationality of the host population. In reality, we cannot neglect the non-stationality to deal with endemic diseases over a long period of time, as the disease dynamics are likely influenced by the host age-structure and growth. Several studies assuming homogeneous patterns of transmission have dealt with growing host population, the basic system of which was usually governed by ordinary differential equations ([40], [41], [47], [50]). In addition, a rigorous series of mathematical studies was reported on the transmission dynamics in a growing population with loss of acquired immunity ([35], [5], [6], [7], [9], [19]). However, except for a few studies ([32], [33], [52], [36], [58]), limited number of epidemic models have taken into account both the age structure of host population and its growth. Explicitly age-duration-structured model assuming the non-stationality of host population have yet to be formulated.

Thus, it is of practical importance to develop a basic theory of an age-structured epidemic system assuming non-stationality of the host populations. In particular, it should be valuable to offer a method to evaluate the basic reproduction number and the relevant indices which can characterize the infectious disease dynamics in non-stationary age-structured host populations. Since the theoretical aspect of this project has been partly realized in [28] and [30], the present study focuses on the basic theory and methods which could activate statistical estimations of $R_{0}$, highlighting the eradication threshold and drawing the impact of population growth rate on the threshold conditions.

In Section 2, we present a basic theory of age-duration-structured SIR epidemic model in 
a stable population. Although this type of age-structured SIR model was first proposed by Dietz and Schenzle more than 20 years before [14], which has continued to be the most standard SIR type structured epidemic model, its mathematical implications have never been exhausted. In Section 3, we formulate the linearized system describing the invasion phase of the epidemic, which is used to define the basic reproduction number. The idea of the effective reproduction number is also discussed to formulate the eradication condition by vaccination. In Section 4, we characterize the endemic steady state employing the effective next generation operator. In Section $5, R_{0}$ with various different assumptions are analytically derived extending previous methods by Dietz and Schenzle [14] and Farrington, Kanaan and Gay [17]. Estimators of $R_{0}$ under various assumptions are offered, which can explicitly account for non-zero population growth rate. As the special cases, simplified estimators are also demonstrated assuming that survivorship of the host population follows either exponential or uniform distribution. In Section 6, key issues in immunization are discussed in relation to the threshold condition presented in previous sections. We examine critical coverage of vaccination which explicitly incorporates population growth rate. In Section 7, various sensitivity analyses are performed as numerical examples. We examine the sensitivity of $R_{0}$ and average age at infection to different values of population growth rate given the same estimate of the force of infection. Moreover, sensitivity of the critical coverage of vaccination is examined in relation to the population growth rate and age to complete mass vaccination. In Section 8, we summarize our findings and discuss important implications. The present study is finalized suggesting future issues to be clarified.

\section{The Homogeneous SIR Epidemic System}

Consider a large scale closed host population with age structure. Let us introduce $I(t, \tau ; a)$ as the density of infected population at time $t$ and infection-age (the time since infection) $\tau$ whose chronological age at infection is $a$. Let $S(t, a)$ be the age density of susceptibles at time $t$ and age $a$ and $R(t, \tau ; a)$ the density of recovered individuals at time $t$, duration (the time elapsed since the recovery) $\tau$ and age at recovery $a$.

Then the age-duration-dependent homogeneous SIR epidemic system ([14], [30]) is for- 
mulated as follows:

$$
\begin{aligned}
\left(\frac{\partial}{\partial t}+\frac{\partial}{\partial a}\right) S(t, a) & =-(\mu(a)+\lambda(t, a)) S(t, a) \\
\left(\frac{\partial}{\partial t}+\frac{\partial}{\partial \tau}\right) I(t, \tau ; a) & =-(\mu(a+\tau)+\gamma(\tau)) I(t, \tau ; a) \\
\left(\frac{\partial}{\partial t}+\frac{\partial}{\partial \tau}\right) R(t, \tau ; a) & =-\mu(a+\tau) R(t, \tau ; a) \\
S(t, 0) & =B(t) \\
I(t, 0 ; a) & =\lambda(t, a) S(t, a) \\
R(t, 0 ; a) & =\int_{0}^{a} \gamma(\tau) I(t, \tau ; a-\tau) d \tau
\end{aligned}
$$

where $B(t)$ is the number of newborns per unit time, $\mu(a)$ the force of mortality, $\gamma(\tau)$ the rate of recovery at infection-age $\tau$ and $\lambda$ is the force of infection (FOI). The FOI is the infection rate of susceptible population per unit time and per capita. We assume that FOI is given by the true mass-action model [10]:

$$
\lambda(t, a)=\frac{1}{N(t)} \int_{0}^{\infty} \beta(a, \sigma) \int_{0}^{\sigma} f(\tau) I(t, \tau ; \sigma-\tau) d \tau d \sigma
$$

where $\beta(a, \sigma)$ can be interpreted as the probability that a susceptible individual with age $a$ encounters with an inflected individuals with age $\sigma$, and the function $f(\tau)$ is the probability of successful transmission of infective agents from infective individuals with infection-age $\tau$, and $N(t)$ is the total size of host population given by

$$
N(t):=\int_{0}^{\infty} P(t, a) d a,
$$

where $P(t, a)$ is the age density of host population:

$$
P(t, a):=S(t, a)+\int_{0}^{a} I(t, a-\tau ; \tau) d \tau+\int_{0}^{a} R(t, a-\tau ; \tau) d \tau .
$$

Moreover we assume that all newborns are susceptible, so $I(t, 0 ; 0)=R(t, 0 ; 0)=0$ and $B(t)=P(t, 0)$, which is given as

$$
B(t)=P(t, 0)=\int_{0}^{\infty} m(a) P(t, a) d a,
$$

where $m(a)$ is the age-specific birth rate. We assume that $m(\cdot)$ is a nonnegative, essentially bounded measurable functions with a compact support.

The key epidemiological assumption here is that the force of infection is proportional to the ratio of infected population to the host population, so it is independent from the scale of 
population. Then the basic system (2.1) becomes a homogeneous system, of which general character has been discussed in [30] and [31].

Then the host population dynamics is described by the Lotka-McKendrick system (the stable population model):

$$
\begin{aligned}
\left(\frac{\partial}{\partial t}+\frac{\partial}{\partial a}\right) P(t, a) & =-\mu(a) P(t, a), \\
P(t, 0) & =\int_{0}^{\infty} m(a) P(t, a) d a,
\end{aligned}
$$

where we assume that demographic birth and death rates are not affected by epidemiological status.

In the following we assume that the host population has already attained a stable population. Hence

$$
P(t, a)=N(t) \psi(a), \quad N(t)=N(0) e^{r_{0} t}
$$

where

$$
\psi(a):=b_{0} e^{-r_{0} a} \ell(a), \quad \ell(a):=\exp \left(-\int_{0}^{a} \mu(\sigma) d \sigma\right)
$$

$\psi$ denotes the normalized stable age distribution, $\ell(a)$ the survival rate with respect to the natural death rate, $r_{0}$ the intrinsic rate of natural increase determined by the real root of the Euler-Lotka equation:

$$
\int_{0}^{\infty} e^{-r_{0} a} m(a) \ell(a) d a=1
$$

and $b_{0}$ is the crude birth rate of the stable population given by

$$
b_{0}=\frac{1}{\int_{0}^{\infty} e^{-r_{0} a} \ell(a) d a} .
$$

Let us introduce new variables $s(t, a)$ and $i(t, \tau ; a)$ by

$$
\begin{aligned}
S(t, a) & =s(t, a) P(t, a), \\
I(t, \tau ; a) & =i(t, \tau ; a) P(t, a+\tau), \\
R(t, \tau ; a) & =r(t, \tau ; a) P(t, a+\tau) .
\end{aligned}
$$

The density $s(t, a)$ denotes the proportion of susceptibles at age $a$ and time $t, i(t, \tau ; a)$ is the proportion of infectives at age $a+\tau$, infection-age $\tau$ and time $t$ and $r(t, \tau ; a)$ denotes the proportion of removed individuals at age $a+\tau$, duration $\tau$ and time $t$. Then it follows from (2.4) that

$$
s(t, a)+\int_{0}^{a} i(t, a-\tau ; \tau) d \tau+\int_{0}^{a} r(t, a-\tau ; \tau) d \tau=1 .
$$

Since the removed class $r(t, \tau ; a)$ is determined by $s$ and $i$, it is sufficient to consider $(s, i)$ system. 
Then the basic system (2.1) is reduced to a normalized system:

$$
\begin{aligned}
\left(\frac{\partial}{\partial t}+\frac{\partial}{\partial a}\right) s(t, a) & =-\lambda(t, a) s(t, a) \\
\left(\frac{\partial}{\partial t}+\frac{\partial}{\partial \tau}\right) i(t, \tau ; a) & =-\gamma(\tau) i(t, \tau ; a) \\
s(t, 0) & =1 \\
i(t, 0 ; a) & =\lambda(t, a) s(t, a) \\
\lambda(t, a) & =\int_{0}^{\infty} \beta(a, \sigma) \psi(\sigma) \int_{0}^{\sigma} f(\tau) i(t, \tau ; \sigma-\tau) d \tau d \sigma
\end{aligned}
$$

Since the original age-duration density functions should be integrable, the state space $\Omega$ of the normalized distribution $(s, i)$ is given by

$$
\Omega:=\left\{(s, i) \in X: 0 \leq s \leq 1,0 \leq i \leq 1, s(a)+\int_{0}^{a} i(\tau ; a-\tau) d \tau \leq 1\right\}
$$

where $X:=L_{\psi}\left(\mathbb{R}_{+}\right) \times E, E:=L^{1}\left(\mathbb{R}_{+} ; L_{\psi}\left(\mathbb{R}_{+}\right)\right)$and $L_{\psi}$ is a weighted $L^{1}$-space defined by

$$
L_{\psi}\left(\mathbb{R}_{+}\right):=\left\{\psi^{-1} \phi \mid \phi \in L^{1}\left(\mathbb{R}_{+}\right)\right\}
$$

with a norm $\|s\|_{L_{\psi}}:=\int_{0}^{\infty}|s(a)| \psi(a) d a$. For $i \in E$, its norm is also defined as

$$
\|i\|_{E}=\int_{0}^{\infty} \int_{0}^{\infty}|i(\tau ; a)| \psi(a) d a d \tau
$$

There are several approaches to show mathematical well-posedness (existence and uniqueness of solutions) of the time evolution problem as (2.1) or (2.13), in particular, the semigroup approach using the theory of non densely defined generator would be most advantageous to prove the well-posedness and the linearized stability ([39], [29]). However, here we sketch a classical integral equation approach to the homogeneous system (2.13) by reducing it to a nonlinear (vector-valued) integral equation, which would be also useful to prove the global stability of the disease-free steady state and to formulate the eradication threshold by vaccination.

Here we introduce technical assumptions, which may not be necessarily essential, but they are biologically reasonable and sufficient to prove the following mathematical conclusions related to abstract renewal equation ([24], [26]).

\section{Assumption 2.1.}

1. There exist positive, essentially bounded functions $\beta_{-}$and $\beta_{+}$such that for all $\sigma \geq 0$,

$$
\beta_{-}(a) \leq \beta(a, \sigma) \leq \beta_{+}(a)
$$


2. The following holds uniformly for $\sigma \geq 0$ :

$$
\lim _{h \rightarrow 0} \int_{0}^{\infty}|\psi(a+h) \beta(a+h, \sigma)-\psi(a) \beta(a, \sigma)| d a=0,
$$

where the domain of $\psi \beta$ is extended as $\psi \beta=0$ for $a<0$.

3. The force of mortality $\mu$ is nonnegative, essentially bounded function and there exist constants $0<\mu_{\infty}<\infty$ and $M_{\mu}>0$ such that

$$
\lim _{a \rightarrow \infty} \mu(a)=\mu_{\infty}, \quad \int_{0}^{\infty}\left|\mu(a)-\mu_{\infty}\right| d a \leq M_{\mu}
$$

4. The infectivity function $f(\tau)$ is a nonnegative, essentially bounded function and there exists an upper bound of the infectious period $L$ such that $f(\tau)=0$ for all $\tau>L$. The recovery rate $\gamma(\tau)$ is nonnegative, locally integrable function.

First we note that the Assumption 2.1-3 is used to guarantee the integrability of the stable distribution $\psi$. In fact it is easy to see that $\psi(a) \leq b_{0} e^{-\left(r_{0}+\mu_{\infty}\right)+M_{\mu}}$, hence $\psi$ becomes integrable.

Lemma 2.2. Under the Assumption 2.1-3, the normal age distribution $\psi$ is bounded and integrable for $r_{0}>-\mu_{\infty}$.

Assumption 2.3. In the following, we assume that $r_{0}>-\mu_{\infty}$.

By solving the McKendrick equation for $i(t, \tau ; a)$ in $(2.13)$ along the characteristic line, it follows that

$$
i(t, \tau ; a)= \begin{cases}\Gamma(\tau) i(t-\tau, 0 ; a), & t-\tau>0, \\ \frac{\Gamma(\tau)}{\Gamma(\tau-t)} i(0, \tau-t ; a), & \tau-t>0,\end{cases}
$$

where $\Gamma(\tau)$ is the survival function induced from the recovery rate $\gamma(\tau)$ given by

$$
\Gamma(\tau):=\exp \left(-\int_{0}^{\tau} \gamma(\sigma) d \sigma\right) .
$$

Let us introduce a new variable $\xi(t, a):=\psi(a) i(t, 0 ; a)=I(t, 0 ; a) / N(t)$. Then we know that $\xi(t, a)$ gives the incidence rate of new infection at age $a$ with respect to the total population size at time $t$ in the original system $(2.1)$, while $i(t, 0 ; a)=I(t, 0 ; a) / P(t, a)$ is the age-specific incidence rate of new infection.

Inserting (2.17) into the expression of $\lambda$ and changing the order of integrals, we have

$$
\begin{aligned}
\xi(t, a) & =\psi(a) s(t, a) \lambda(t, a) \\
& =\psi(a) s(t, a)\left[g(t, a)+\int_{0}^{t} d \tau \int_{\tau}^{\infty} \beta(a, \sigma) \psi(\sigma) f(\tau) \Gamma(\tau) i(t-\tau, 0 ; \sigma-\tau) d \sigma\right] \\
& =\psi(a) s(t, a)\left[g(t, a)+\int_{0}^{t} d \tau \int_{0}^{\infty} \beta(a, \tau+\zeta) \frac{\psi(\tau+\zeta)}{\psi(\zeta)} f(\tau) \Gamma(\tau) \xi(t-\tau, \zeta) d \zeta\right]
\end{aligned}
$$


where

$$
g(t, a):=\int_{t}^{\infty} d \tau \int_{\tau}^{\infty} \beta(a, \sigma) \psi(\sigma) f(\tau) \frac{\Gamma(\tau)}{\Gamma(\tau-t)} i(0, \tau-t ; \sigma-\tau) d \sigma .
$$

Since we can see $\xi(t)(\cdot):=\xi(t, \cdot)$ as a function from $\mathbb{R}_{+}$to $L^{1}$, the equation $(2.18)$ can be seen as an abstract integral equation in $L^{1}$ :

$$
\xi(t)=\psi s(t)\left[g(t)+\int_{0}^{t} \Psi(\tau) \xi(t-\tau) d \tau\right]
$$

where $\Psi(\tau)$ is a nonnegative operator from $L_{+}^{1}$ into $L_{+}^{\infty}$ defined by

$$
(\Psi(\tau) \xi)(a):=\int_{0}^{\infty} \beta(a, \tau+\zeta) \frac{\psi(\tau+\zeta)}{\psi(\zeta)} f(\tau) \Gamma(\tau) \xi(\zeta) d \zeta, \quad \xi \in L^{1}\left(\mathbb{R}_{+}\right) .
$$

Again let us introduce a new function $u(t, a):=\psi(a)(1-s(t, a))$. Then $u(t, \cdot)$ is a $L_{1}$-valued function and $u$ is a solution of the following system:

$$
\begin{aligned}
\left(\frac{\partial}{\partial t}+\frac{\partial}{\partial a}\right) u(t, a) & =-\left(r_{0}+\mu(a)\right) u(t, a)+\xi(t, a) \\
u(t, 0) & =0 .
\end{aligned}
$$

In the abstract setting, the above system can be seen as a Cauchy problem in $L^{1}$ :

$$
\frac{d u(t)}{d t}=A u(t)+\xi(t)
$$

where $A$ is the population operator defined by

$$
(A \phi)(a):=-\frac{d \phi(a)}{d a}-\left(r_{0}+\mu(a)\right) \phi(a), \quad \phi \in D(A)
$$

with the domain $D(A)$ given by $D(A)=\left\{\phi \mid \phi \in W^{1,1}(\mathbb{R}), \phi(0)=0\right\}$.

Since it is well-known that the population operator $A$ generates a $C_{0}$-semigroup $e^{t A}$ in $L^{1}$ ([55], [56]), it follows from the variation of constants formula that

$$
u(t)=e^{t A} u(0)+\int_{0}^{t} e^{(t-\sigma) A} \xi(\sigma) d \sigma .
$$

Let us introduce continuous functions $x_{1}(t)$ from $\mathbb{R}_{+}$to $L^{1}\left(\mathbb{R}_{+}\right)$and $x_{2}(t)$ from $\mathbb{R}_{+}$to $L^{\infty}\left(\mathbb{R}_{+}\right)$by

$$
x_{1}(t):=\psi-u(t), \quad x_{2}(t):=g(t)+\int_{0}^{t} \Psi(\tau) \xi(t-\tau) d \tau .
$$

From $x_{1}=\psi s$ and (2.19), we have $\xi(t)=x_{1}(t) x_{2}(t)$ and

$$
\begin{aligned}
& x_{1}(t)=\psi-e^{t A} u(0)-\int_{0}^{t} e^{\tau A} x_{1}(t-\tau) x_{2}(t-\tau) d \tau, \\
& x_{2}(t)=g(t)+\int_{0}^{t} \Psi(\tau) x_{1}(t-\tau) x_{2}(t-\tau) d \tau .
\end{aligned}
$$


Then we arrive at a nonlinear renewal integral equation of vector-valued continuous function $z \in C\left(\mathbb{R}_{+} ; L^{1}\left(\mathbb{R}_{+}\right) \times L^{\infty}\left(\mathbb{R}_{+}\right)\right)$as

$$
z(t)=h(t)+\int_{0}^{t} \Phi(\tau) f(z(t-\tau)) d \tau
$$

where

$$
\begin{gathered}
z(t):=\left(\begin{array}{l}
x_{1}(t) \\
x_{2}(t)
\end{array}\right), \quad h(t):=\left(\begin{array}{c}
\psi-e^{t A} u(0) \\
g(t)
\end{array}\right), \\
\Phi(\tau):=\left(\begin{array}{cc}
-e^{\tau A} & 0 \\
0 & \Psi(\tau)
\end{array}\right), \quad f(z(t)):=\left(\begin{array}{l}
x_{1}(t) x_{2}(t) \\
x_{1}(t) x_{2}(t)
\end{array}\right) .
\end{gathered}
$$

By using the standard fixed point argument, it is not difficult to show that the integral equation (2.26) has a unique continuous solution for $t \in[0, \infty)$ (see [44], chapter VI). Then $s(t)=\psi^{-1} x_{1}(t)$ and $i(t, \tau ; a)$ is determined by $(2.17)$ using $i(t, 0 ; a)=\psi^{-1}(a) x_{1}(t)(a) x_{2}(t)(a)$.

From (2.17) and (2.21), we know that $s(t, a)$ and $i(t, \tau ; a)$ has a directional derivative $D$ defined by

$$
D \phi(t, \tau):=\lim _{h \rightarrow 0} \frac{\phi(t+h, \tau+h)-\phi(t, \tau)}{h} .
$$

Then we conclude that if the derivative $\partial_{t}+\partial_{\tau}$ is interpreted as the directional derivative $D$, the basic system (2.1) formulate a mathematically well-posed problem [25].

\section{Linear Invasion Process and the Definition of $R_{0}$}

Now let us consider a situation (disease invasion phase) that very few infected individuals appear in the completely susceptible host population. Then the dynamics of the infected population is described by the linearized equation at the disease-free steady state $(s, i)=$ $(1,0)$ given by

$$
\begin{aligned}
\left(\frac{\partial}{\partial t}+\frac{\partial}{\partial \tau}\right) i(t, \tau ; a) & =-\gamma(\tau) i(t, \tau ; a) \\
i(t, 0 ; a) & =\int_{0}^{\infty} \beta(a, \sigma) \psi(\sigma) \int_{0}^{\sigma} f(\tau) i(t, \tau ; \sigma-\tau) d \tau d \sigma .
\end{aligned}
$$

Let $\chi(t, a):=\psi(a) i(t, 0 ; a)$ be the incidence rate (for new infection at age $a$ ) at time $t$ for the linearized system. By solving the McKendrick equation (3.1) for $i(t, \tau ; a)$ along the characteristic line, we again obtain the expression (2.17). Inserting the expression (2.17) into the boundary condition of (3.1) and changing the order of integrals, we have

$$
\chi(t, a)=k(t, a)+\psi(a) \int_{0}^{t} d \tau \int_{0}^{\infty} \beta(a, \tau+\zeta) \frac{\psi(\tau+\zeta)}{\psi(\zeta)} f(\tau) \Gamma(\tau) \chi(t-\tau, \zeta) d \zeta,
$$

where

$$
k(t, a)=\int_{0}^{\infty} d \tau \int_{0}^{\infty} \psi(a) \beta(a, t+\zeta+\tau) \psi(t+\zeta+\tau) f(t+\tau) \frac{\Gamma(t+\tau)}{\Gamma(\tau)} i(0, \tau ; \zeta) d \zeta .
$$


Then the equation (3.2) can be regarded as an abstract renewal equation in $L^{1}$ :

$$
\chi(t)=k(t)+\int_{0}^{t} \Theta(\tau) \chi(t-\tau) d \tau .
$$

where $k(t)(a)=k(t, a), \chi(t)(a)=\chi(t, a)$ and $\Theta(\tau):=\psi \Psi(\tau)$.

Then $\Theta(\tau)$ defines a nonnegative operator from $L_{+}^{1}$ into itself. Moreover, it follows from the well-known compactness criterion in $L^{1}$ that the Assumption 2.1-2 makes $\Theta(\tau)$ a compact operator. Using the Assumption 2.1-4, we can easily prove the following estimates:

Lemma 3.1. There exists a number $M_{g}>0$ such that $\|k(t)\|_{L^{1}} \leq M_{g} e^{-\left(r_{0}+\mu_{\infty}\right) t}\left\|i_{0}\right\|_{E}$.

From Lemma 3.1, the Laplace transform of $k(t)$, denoted by $\hat{k}(z):=\int_{0}^{\infty} e^{-z t} k(t) d t$, exists for $\Re z>-\left(r_{0}+\mu_{\infty}\right)$.

Lemma 3.2. Under the Assumption 2.1, the Laplace transform of $\Theta(\tau)$, denoted by $\hat{\Theta}(z):=$ $\int_{0}^{\infty} e^{-z \tau} \Theta(\tau) d \tau$, exists for all $z \in \mathbb{C}$ and $\hat{\Theta}(z)$ is a nonsupporting operator ${ }^{2}$ on the cone $L_{+}^{1}$, its spectral radius $r(\hat{\Theta}(z))$ is monotone decreasing with respect to $z$ and $\lim _{z \rightarrow \infty} r(\hat{\Pi}(z))=0$. Proof. From the Assumption 2.1-1, we have

$$
<F, \phi>\psi \beta_{-} \leq \hat{\Theta}(z) \phi \leq e^{M_{\mu}}<F, \phi>\psi \beta_{+},
$$

where $F$ is a strictly positive functional defined by

$$
<F, \phi>:=\int_{0}^{L} e^{-\left(z+r_{0}+\mu_{\infty}\right) \tau} f(\tau) \Gamma(\tau) d \tau\|\phi\|_{L^{1}},
$$

for $\phi \in L^{1}\left(\mathbb{R}_{+}\right)$. Therefore $\hat{\Theta}(\tau)$ is well defined for all $z \in \mathbb{C}$. For real $z$, we obtain that for integer $n$

$$
\hat{\Theta}(z)^{n} \phi \geq<F, \phi><F, \psi \beta_{-}>^{n-1} \psi \beta_{-} .
$$

Then for any nonzero nonnegative functional $G$ of the dual cone of $L_{+}^{1}$ and $\phi \in L_{+}^{1} \backslash\{0\}$, we have $<G, \hat{\Theta}^{n}(z) \phi>>0$, which shows that $\hat{\Theta}(z)$ is nonsupporting in the sense of Sawashima [48]. From the comparison theorem [38], $r(\hat{\Theta}(z))$ is monotone decreasing for $z \in \mathbb{R}$. From the estimate (3.5), it is clear that $\lim _{z \rightarrow \infty} r(\hat{\Theta}(z))=0$.

Let $\chi_{n}(t)$ be the $n$-th generation of incidence rate at time $t$. Then $k(t)$ is the first generation directly transmitted from the initial infected population (primary cases) and so we can define the successive generations as follows:

$$
\chi_{1}(t)=k(t), \quad \chi_{n+1}(t)=\int_{0}^{t} \Theta(\tau) \chi_{n}(t-\tau) d \tau, \quad n=1,2, \ldots,
$$

Then the total size of $n$-th generation of the incidence rate is calculated iteratively as follows:

$$
\begin{aligned}
\int_{0}^{\infty} \chi_{n+1}(t) d t & =\int_{0}^{\infty} \int_{0}^{t} \Theta(\tau) \chi_{n}(t-\tau) d \tau d t \\
& =\int_{0}^{\infty} \Theta(\tau) d \tau \int_{0}^{\infty} \chi_{n}(t) d t, \quad(n \geq 1) .
\end{aligned}
$$

\footnotetext{
${ }^{2}$ For the definition of nonsupporting operators, the reader may refer to [24] and [26].
} 
Therefore the total size of $n$-th generation of the incidence rate, denoted by $X_{n}:=\int_{0}^{\infty} \chi_{n}(t) d t$ is calculated as

$$
X_{n+1}=K^{n} X_{1}
$$

where the iteration operator

$$
K:=\hat{\Theta}(0)=\int_{0}^{\infty} \Theta(\tau) d \tau
$$

acting on $L^{1}\left(\mathbb{R}_{+}\right)$is given by

$$
(K \phi)(a):=\psi(a) \int_{0}^{\infty} \int_{0}^{\infty} \beta(a, \zeta+\tau) \frac{\psi(\zeta+\tau)}{\psi(\zeta)} f(\tau) \Gamma(\tau) d \tau \phi(\zeta) d \zeta, \quad \phi \in L^{1}\left(\mathbb{R}_{+}\right) .
$$

The spectral radius of the linear operator $K$ is defined by

$$
r(K)=\lim _{n \rightarrow \infty} \sqrt[n]{\left\|K^{n}\right\|}
$$

Hence we know from (3.9) and (3.12) that the spectral radius $r(K)$ gives the asymptotic growth factor of the generation size $X_{n}$.

If $K$ is a nonsupporting compact operator, $r(K)$ becomes the positive dominant (Frobenius) eigenvalue of $K$, that is, $|\mu|<r(K)$ for any eigenvalue $\mu$ of $K$ different from $r(K)$. Moreover there exists a one-dimensional projection operator $P$ such that

$$
\lim _{n \rightarrow \infty} r(K)^{-n} K^{n} \phi=P \phi, \quad \phi \in L^{1}
$$

where $P$ is the projection to the eigenspace spanned by the positive eigenvector $u_{0}$ corresponding to the eigenvalue $r(K)$ ([37], [38]). In fact, we can prove that

$$
P \phi=\frac{<F_{0}, \phi>}{<F_{0}, u_{0}>} u_{0}
$$

where $F_{0}$ is the eigenfunctional of the dual operator $K^{*}$ associated with the Frobenius eigenvalue $r(K)$.

That is, $r(K)$ gives the asymptotic growth factor of the $n$-th generation size (vector) $X_{n}$ and it becomes asymptotically proportional to the Frobenius eigenvector $u_{0}$ of $K$ :

$$
K^{n} \phi \sim r(K)^{n} \frac{<F_{0}, \phi>}{<F_{0}, u_{0}>} u_{0}, \quad(t \rightarrow \infty) .
$$

The basic reproduction number $R_{0}$ is defined as the spectral radius of the next generation operator, which maps the density of primary cases to the density of secondary cases [11]. From the above argument, we can conclude that the next generation operator of our homogeneous model (2.1) is given by the positive integral operator $K$ and $R_{0}=r(K)$.

Though $R_{0}$ gives the asymptotic growth factor of the generation size of the infecteds (in our case, it is the incidence rate), we still have to establish a relation to the intrinsic growth 
rate of the infected population in real time. We should remark that here the intrinsic growth rate refers to the relative growth rate of infected population in real time, since the system (2.13) is a normalized system by (2.11). In fact, if $z_{0}$ is the growth rate in the normalized system as $i(t, \tau ; a)=e^{z_{0} t} i(0, \tau ; a)$, then $I(t, \tau ; a)=e^{z_{0} t} i(0, \tau ; a) N(0) e^{r_{0} t} \psi(a+\tau)$, so the growth rate is $r_{0}+z_{0}$ in the real population.

From Lemma 3.2 and results by Sawashima [48] and Marek [38], the Perron-Frobenius property holds for nonsupporting compact operators, hence its spectral radius $r(\hat{\Theta}(z))$ is the positive dominant eigenvalue and monotone decreasing function for $z \in \mathbb{R}$. Since $r(\hat{\Theta}(z))$ decreases from $\lim _{z \rightarrow-\left(r_{0}+\mu_{\infty}\right)} r(\hat{\Theta}(z))$ to zero as $z$ moves from $-\left(r_{0}+\mu_{\infty}\right)$ to $+\infty$, there exists a unique root $z_{0}>-\left(r_{0}+\mu_{\infty}\right)$ such that $r(\hat{\Theta}(z))=1$ if $\lim _{z \rightarrow-\left(r_{0}+\mu_{\infty}\right)} r(\hat{\Theta}(z))>1$. As $R_{0}=r(\hat{\Theta}(0))$ and we assume $r_{0}+\mu_{\infty}>0$, we conclude that $z_{0}>0$ if $R_{0}>1, z_{0}=0$ if $R_{0}=1$ and $-\left(r_{0}+\mu_{\infty}\right)<z_{0}<0$ if $R_{0}<1<\lim _{z \rightarrow-\left(r_{0}+\mu_{\infty}\right)} r(\hat{\Theta}(z))$. In those cases it is proved that

$$
\lim _{t \rightarrow \infty} e^{-z_{0} t} \chi(t)=\frac{<F_{z_{0}}, \hat{k}\left(z_{0}\right)>}{<F_{z_{0}}, u_{z_{0}}>} u_{z_{0}},
$$

where $u_{z_{0}}$ is the eigenvector corresponding to the eigenvalue unity of $\hat{\Theta}\left(z_{0}\right)$ and $F_{z_{0}}$ is its dual eigenfunctional. Even for the case that $R_{0}<\lim _{z \rightarrow-\left(r_{0}+\mu_{\infty}\right)} r(\hat{\Theta}(z))<1$, from a classical stability result for the renewal equation [25] and $\lim _{t \rightarrow \infty} k(t)=0$, we can conclude that $\lim _{t \rightarrow \infty} \chi(t)=0$. Then we can summarize the above argument as the following general principle [11]:

Proposition 3.5. [Invasion Threshold Principle] Let $R_{0}$ be the basic reproduction number given as the dominant positive eigenvalue (the spectral radius) of the next generation operator $K$. The disease can invade into the completely susceptible host population if $R_{0}>1$, whereas it cannot if $R_{0}<1$. The intrinsic growth rate for the initial invasion is given by the real root of the characteristic equation $r(\hat{\Theta}(z))=1$.

If we adopt the well-known proportionate mixing assumption (PMA) such that the contact coefficient $\beta$ is factorized as $\beta(a, \sigma)=\beta_{1}(a) \beta_{2}(\sigma)$, which implies that there is no correlation between the age of susceptibles and the age of infectives when contacts occur, the eigenfunction $u_{z_{0}}$ is then proportional to $\psi \beta_{1}$ and the eigenvalue equation $\hat{\Theta}\left(z_{0}\right) u_{z_{0}}=u_{z_{0}}$ is reduced to the following characteristic equation:

$$
\int_{0}^{\infty} \int_{0}^{\infty} e^{-z_{0} \tau} \beta_{2}(\tau+\zeta) \psi(\tau+\zeta) f(\tau) \Gamma(\tau) \beta_{1}(\zeta) d \zeta d \tau=1
$$

Then in this PMA case, it is easy to see that there exists a unique $r_{0} \in \mathbb{R}$ satisfying the characteristic equation (3.17).

Here we should also remark that the stability of the disease-free steady state guaranteed by $R_{0}<1$ is local linearized stability. In fact, for our case $R_{0}<1$ implies not only the linearized stability of the disease-free steady state, but also its global stability. To see this fact, again consider the nonlinear epidemic process. Since $s(t, a) \leq 1$, it follows from $(2.19)$ 
that

$$
\xi(t) \leq k(t)+\int_{0}^{t} \Theta(\tau) \xi(t-\tau) d \tau
$$

¿From the comparison argument, we have $\xi(t) \leq \chi(t)$. Since $\lim _{t \rightarrow \infty} \chi(t)=0$ if $R_{0}<1$, we conclude that

Proposition 3.6. If $R_{0}<1$, the disease-free steady state is globally asymptotically stable.

Suppose that a part of newborns are vaccinated only once during the very early infancy. Let $\epsilon \in[0,1]$ be the ratio of vaccinated newborns. Then the boundary condition for $s$ in (2.13) is replaced by $s(t, 0)=1-\epsilon$, so the effective reproduction number, denoted by $R_{e}{ }^{3}$ is given by $R_{e}=(1-\epsilon) R_{0}$ and the epidemic is eradicated if and only if $R_{e}<1$, that is, the vaccination ratio satisfies

$$
\epsilon>1-\frac{1}{R_{0}} .
$$

Define the effective next generation operator (ENGO) $K_{e}$ by $K_{e}:=(1-\epsilon) K$. Suppose that $R_{e}=r\left(K_{e}\right)<1$. If the vaccination is introduced, (3.22) is rewritten as

$$
\xi(t) \leq(1-\epsilon) k(t)+(1-\epsilon) \int_{0}^{t} \Theta(\tau) \xi(t-\tau) d \tau
$$

If we take any large positive number $t_{0}>0$ to integrate $\xi(t)$ from 0 to $t_{0}$, we have

$$
\begin{aligned}
\int_{0}^{t_{0}} \xi(t) d t & \leq(1-\epsilon) \int_{0}^{t_{0}} k(t) d t+(1-\epsilon) \int_{0}^{t_{0}} d t \int_{0}^{t} \Theta(\tau) \xi(t-\tau) d \tau \\
& \leq(1-\epsilon) \int_{0}^{\infty} k(t) d t+(1-\epsilon) \int_{0}^{\infty} \Theta(\tau) d \tau \int_{0}^{t_{0}} \xi(t) d t
\end{aligned}
$$

Therefore if the eradication condition (3.19) is satisfied, that is, $R_{e}<1, I-K_{e}$ is nonnegatively invertible and we have

$$
\int_{0}^{t_{0}} \xi(t) d t \leq(1-\epsilon)\left(I-K_{e}\right)^{-1} \int_{0}^{\infty} k(t) d t
$$

which shows that $\int_{0}^{\infty} \xi(t) d t$ exists and

$$
\int_{0}^{\infty} \xi(t) d t \leq(1-\epsilon)(I-(1-\epsilon) K)^{-1} \int_{0}^{\infty} k(t) d t
$$

Since $\xi(t, a)$ denotes the incidence rate, we conclude that if $R_{e}<1$, which case is called a small (subcritical) epidemic, the total size of incidence is less than

$$
(1-\epsilon)\left\|\left(I-K_{e}\right)^{-1} \int_{0}^{\infty} k(t) d t\right\|_{L^{1}} .
$$

\footnotetext{
${ }^{3}$ If the host population is not necessarily completely susceptible, the corresponding reproduction number is referred to as the effective reproduction number.
} 


\section{Endemic Threshold and Stationarity}

Let us consider the reproduction process of infected population at the endemic steady state. Stationary solutions of the normalized system, which corresponds to persistent solutions of the homogeneous system, indicated by asterisk, satisfy the following system:

$$
\begin{aligned}
\frac{d s^{*}(a)}{d a} & =-\lambda^{*}(a) s^{*}(a), \\
\frac{\partial i(\tau ; a)}{\partial \tau} & =-\gamma(\tau) i^{*}(\tau ; a), \\
s^{*}(0) & =1 \\
i^{*}(0 ; a) & =\lambda^{*}(a) s^{*}(a), \\
\lambda^{*}(a) & =\int_{0}^{\infty} \beta(a, \sigma) \psi(\sigma) \int_{0}^{\sigma} f(\tau) i^{*}(\tau ; \sigma-\tau) d \tau d \sigma,
\end{aligned}
$$

where $\lambda^{*}(a)$ denotes the force of infection at the endemic steady state. Then we have

$$
\begin{aligned}
s^{*}(a) & =e^{-\int_{0}^{a} \lambda^{*}(\sigma) d \sigma}, \\
i^{*}(\tau ; a) & =\lambda^{*}(a) s^{*}(a) \Gamma(\tau),
\end{aligned}
$$

where we note that $s^{*}(a)$ gives the survival rate associated with the force of infection $\lambda^{*}(a)$, which could be obtained from age-specific serological survey data of the endemic disease.

Inserting (4.2) into the expression of FOI in (4.1), we obtain an integral equation for the FOI at the endemic steady state:

$$
\begin{aligned}
\lambda^{*}(a) & =\int_{0}^{\infty} \beta(a, \sigma) \psi(\sigma) \int_{0}^{\sigma} f(\tau) \Gamma(\tau) \lambda^{*}(\sigma-\tau) s^{*}(\sigma-\tau) d \tau d \sigma \\
& =\int_{0}^{\infty} \int_{0}^{\infty} \beta(a, \zeta+\tau) \psi(\zeta+\tau) f(\tau) \Gamma(\tau) d \tau \lambda^{*}(\zeta) s^{*}(\zeta) d \zeta .
\end{aligned}
$$

Therefore if we define the incidence of new infection at the endemic steady state by

$$
\xi^{*}(a):=\lambda^{*}(a) s^{*}(a) \psi(a),
$$

it satisfies the following equation:

$$
\xi^{*}(a)=s^{*}(a) \psi(a) \int_{0}^{\infty} \int_{0}^{\infty} \beta(a, \zeta+\tau) \frac{\psi(\zeta+\tau)}{\psi(\zeta)} f(\tau) \Gamma(\tau) d \tau \xi^{*}(\zeta) d \zeta .
$$

Using the next generation operator $K$, we can formulate (4.5) as

$$
\xi^{*}(a)=s^{*}(a)\left(K \xi^{*}\right)(a) .
$$

Then if we define $T:=s^{*} K$ as the effective next generation operator at the endemic steady state (ENGO at ESS), the condition (4.6) implies that

$$
r(T)=1,
$$


and $\xi^{*}$ is the positive (Frobenius) eigenvector of $T$ associated with eigenvalue unity. The reproduction number $R_{\text {ess }}=r(T)$ is the effective reproduction number at the endemic steady state. The condition (4.7) reflects the fact that, at equilibrium, each infection will on average produce exactly one secondary infection.

Since $K$ is a compact nonsupporting operator, then if $R_{0} \leq 1$, we have $r(T)<r(K)=$ $R_{0} \leq 1$, which contradicts (4.7). Hence we conclude that there is no endemic steady state if $R_{0} \leq 1$. Though we omit the proof, by using a fixed point argument, we can show that if $R_{0}>1$, there is at least one endemic steady state ([26], [30]).

\section{Estimation of $R_{0}$}

From (4.6), we also obtain

$$
\left(K \xi^{*}\right)(a)=\frac{\xi^{*}(a)}{s^{*}(a)}=\psi(a) \lambda^{*}(a)
$$

Let $K^{*}$ be the adjoint operator of $K$ and $F_{0}$ be its eigenfunctional associated with $R_{0}$. Observe that

$$
<K^{*} F_{0}, \xi^{*}>=R_{0}<F_{0}, \xi^{*}>=<F_{0}, K \xi^{*}>
$$

then we have

$$
R_{0}=\frac{<F_{0}, K \xi^{*}>}{<F_{0}, \xi^{*}>}=\frac{<F_{0}, \psi \lambda^{*}>}{<F_{0}, \psi \lambda^{*} s^{*}>} .
$$

The estimation formula (5.2) is an extension of the formula given by Dietz and Schenzle [14] and Farrington, Kanaan and Gay (FKG) [17] for the case that the host population is in a demographic steady state.

Under the proportionate mixing assumption $\beta(a, \sigma)=\beta_{1}(a) \beta_{2}(\sigma)$, it follows that the force of infection (FOI) $\lambda^{*}$ at the endemic steady state is proportional to the susceptibility parameter $\beta_{1}(a)$ :

$$
\lambda^{*}(a)=\beta_{1}(a)<F_{0}, \xi^{*}>.
$$

In particular, note that the FOI is constant if the transmission rate $\beta$ is independent of the age of susceptibles.

Moreover, if the PMA holds, the next generation operator $K$ becomes a one-dimensional operator as

$$
\begin{aligned}
(K \phi)(a) & =\psi(a) \beta_{1}(a) \int_{0}^{\infty} \int_{0}^{\infty} \beta_{2}(\tau+\zeta) \frac{\psi(\tau+\zeta)}{\psi(\zeta)} f(\tau) \Gamma(\tau) d \tau \phi(\zeta) d \zeta \\
& =<F_{0}, \phi>\beta_{1}(a) \psi(a)
\end{aligned}
$$

where $F_{0}$ is the eigenfunctional of the dual operator $K^{*}$ associated with eigenvalue $R_{0}$ given by

$$
<F_{0}, \phi>:=\int_{0}^{\infty} \int_{0}^{\infty} \beta_{2}(\tau+\zeta) \frac{\psi(\tau+\zeta)}{\psi(\zeta)} f(\tau) \Gamma(\tau) d \tau \phi(\zeta) d \zeta
$$


for $\phi \in L^{1}\left(\mathbb{R}_{+}\right)$.

Therefore we know that $\beta_{1} \psi$ is the eigenvector of $K$ associated with $R_{0}$ and it follows that

$$
\begin{aligned}
R_{0} & =<F_{0}, \beta_{1} \psi> \\
& =\int_{0}^{\infty} \int_{0}^{\infty} \beta_{2}(\tau+\zeta) \psi(\tau+\zeta) f(\tau) \Gamma(\tau) d \tau \beta_{1}(\zeta) d \zeta .
\end{aligned}
$$

If we insert the relation (5.3) to (4.3), we know that the coefficient $c:=<F_{0}, \xi^{*}>$ should be given by a positive root of the characteristic equation:

$$
\int_{0}^{\infty} \int_{0}^{\infty} \beta_{2}(\tau+\zeta) \psi(\tau+\zeta) f(\tau) \Gamma(\tau) d \tau \beta_{1}(\zeta) e^{-c \int_{0}^{\zeta} \beta_{1}(\sigma) d \sigma} d \zeta=1
$$

The left hand side of (5.7) gives $R_{0}$ if $c=0$, hence it is easy to see that under the PMA, there exists a unique endemic steady state if and only if $R_{0}>1$.

If we apply the formula (5.2) to the PMA case, it follows from (5.3) that

$$
\begin{aligned}
R_{0} & =\frac{<F_{0}, \psi \lambda^{*}>}{<F_{0}, \psi \lambda^{*} s^{*}>}=\frac{<F_{0}, \psi \beta_{1}>}{<F_{0}, \psi \beta_{1} s^{*}>} \\
& =\frac{\int_{0}^{\infty} \int_{0}^{\infty} \beta_{2}(\tau+\zeta) \psi(\tau+\zeta) f(\tau) \Gamma(\tau) d \tau \beta_{1}(\zeta) d \zeta}{\int_{0}^{\infty} \int_{0}^{\infty} \beta_{2}(\tau+\zeta) \psi(\tau+\zeta) f(\tau) \Gamma(\tau) d \tau \beta_{1}(\zeta) s^{*}(\zeta) d \zeta}
\end{aligned}
$$

In this case, ENGO at ESS is given by

$$
(T \phi)(a)=<F_{0}, \phi>s^{*}(a) \psi(a) \beta_{1}(a)
$$

and its spectral radius must be unity:

$$
R_{\text {ess }}=<F_{0}, \beta_{1} \psi s^{*}>=1,
$$

which is the condition for endemic stationarity. Therefore it follows from (5.10) that the formula (5.8) is reduced to the expression (5.6). However the formula (5.8) is advantageous for estimation purpose, because we can expect that unknown common factors are canceled from its numerator and denominator.

According to Dietz and Schenzle [14], we assume that the length of infectious period $L$ is sufficiently short compared with the life expectancy of host population. Then we obtain

$$
\int_{0}^{\infty} \beta_{2}(\tau+\zeta) \psi(\tau+\zeta) f(\tau) \Gamma(\tau) d \tau \approx \beta_{2}(\zeta) \psi(\zeta) D
$$

where $D$ is the total infectivity of an infected individual given by

$$
D:=\int_{0}^{L} f(\tau) \Gamma(\tau) d \tau
$$


If we use the above approximation in (5.8), we arrive at the estimate:

$$
R_{0} \approx \frac{\int_{0}^{\infty} \beta_{1}(\zeta) \beta_{2}(\zeta) \psi(\zeta) d \zeta}{\int_{0}^{\infty} \beta_{1}(\zeta) \beta_{2}(\zeta) s^{*}(\zeta) \psi(\zeta) d \zeta}
$$

Moreover if we assume that $\beta_{2}(\zeta)$ is proportional to $\beta_{1}(\zeta)$, then $\beta_{1}$ and $\beta_{2}$ are both proportional to $\lambda^{*}$ and we have an estimate:

$$
R_{0} \approx \frac{\int_{0}^{\infty} \lambda^{* 2}(\zeta) \psi(\zeta) d \zeta}{\int_{0}^{\infty} \lambda^{* 2}(\zeta) s^{*}(\zeta) \psi(\zeta) d \zeta},
$$

which are given by Dietz and Schenzle [14] and Greenhalgh and Dietz [21] for the case that the host population is in a demographic steady state.

If we assume that $\beta_{2}$ is constant, we can obtain another useful formula:

$$
R_{0} \approx \frac{\int_{0}^{\infty} \lambda^{*}(\zeta) \psi(\zeta) d \zeta}{\int_{0}^{\infty} \xi^{*}(\zeta) d \zeta}
$$

where we apply (5.3) to (5.11). If the incidence $\xi^{*}$ is observed, we can calculate the FOI as

$$
\lambda^{*}(a)=\frac{\xi^{*}(a)}{\psi(a)\left(1-\int_{0}^{a} \frac{\xi^{*}(\zeta)}{\psi(\zeta)} d \zeta\right)} .
$$

Then $R_{0}$ can be estimated from data of the incidence rate $\xi^{*}$ by using (5.13).

If the force of mortality is constant, the survival rate is given by $\ell(a)=e^{-\mu a}$ ( $\mu$ is a constant force of mortality) and it is referred to as Type II in epidemiology, while if the survival rate is a step function, that is, there exists a maximal age $\omega$ such that $\ell(a)=1$ for $a \in[0, \omega)$ and $\ell(a)=0$ for $a \geq \omega$, then it is referred to as Type $I$ (which implies that the incidence is given by a delta function; $\mu(a) \ell(a)=\delta(a-\omega))$. If we use Type I survival rate, we can establish the final size equation as follows:

Proposition 5.1. Suppose that $\beta_{2}$ is constant and the survival rate is Type I. If the host population is in a demographic stationary state, it follows that

$$
R_{0} \approx-\frac{\log (1-p)}{p}
$$

where $p:=1-s^{*}(\omega)$ is the proportion of ever removed population in a birth cohort.

Proof. If we apply a Type I survival rate such that $\ell(a)=1$ for $a \in[0, \omega)$ and $\ell(a)=0$ for $a \geq \omega$ to (5.13), we have

$$
R_{0} \approx \frac{\int_{0}^{\omega} \lambda^{*}(\zeta) e^{-r_{0} \zeta} d \zeta}{\int_{0}^{\omega} \lambda^{*}(\zeta) s^{*}(\zeta) e^{-r_{0} \zeta} d \zeta} .
$$

If the host population is in a demographic stationary state $\left(r_{0}=0\right)$, it follows that

$$
R_{0} \approx \frac{1}{1-s^{*}(\omega)} \int_{0}^{\omega} \lambda^{*}(\zeta) d \zeta
$$


where $s^{*}(\omega)$ is the proportion of susceptible population at the end of life. From $p=1-s^{*}(\omega)$ and $s^{*}(\omega)=\exp \left(-\int_{0}^{\omega} \lambda^{*}(\zeta) d \zeta\right)$, it follows that

$$
\int_{0}^{\omega} \lambda^{*}(\zeta) d \zeta=-\log (1-p)
$$

Then the estimate (5.15) follows from (5.17) immediately.

The number $p$ can be interpreted as the final size of epidemic with respect to a birth cohort. At individual level, $p$ is called quantum of (infection) event in demographic terminology, which is the lifelong average number of occurrence of infection.

From (5.15), we know that in this case, the well-known final size equation

$$
1-p=e^{-p R_{0}},
$$

holds approximately. However (5.16) shows that the estimate (5.15) for $R_{0}$ would not necessarily be appropriate for non-stationary host populations.

From the expression (5.11), it is easy to see that under appropriate conditions, $R_{0}$ can be computed without knowledge of the transmission rate as follows:

Proposition 5.2. Suppose that the transmission rate $\beta$ is assumed to be constant and the average duration of infectiousness $L$ is short enough. Then it follows that

$$
R_{0} \approx \frac{1}{\int_{0}^{\infty} s^{*}(\zeta) \psi(\zeta) d \zeta}=\frac{1}{x^{*}}
$$

where

$$
x^{*}:=\int_{0}^{\infty} \psi(\zeta) s^{*}(\zeta) d \zeta=b_{0} \int_{0}^{\infty} e^{-\left(r_{0}+\lambda^{*}\right) a} \ell(a) d a,
$$

is the proportion of susceptible population in the host population at the endemic steady state.

That is, we know that the well-known reciprocal relation (5.19) between $R_{0}$ and the proportion of susceptible population at the endemic steady state holds also for the case that the host population is a stable population as far as $\beta$ is constant.

Now let us define $A$ as the average age at the occurrence of new infection:

$$
A:=\frac{\int_{0}^{\infty} a \lambda(t, a) S(t, a) d a}{\int_{0}^{\infty} \lambda(t, a) S(t, a) d a} .
$$

Though in general $A$ is time-dependent, it becomes a constant if the system is on the endemic persistent solution:

$$
A=\frac{\int_{0}^{\infty} a \lambda^{*}(a) s^{*}(a) \psi(a) d a}{\int_{0}^{\infty} \lambda^{*}(a) s^{*}(a) \psi(a) d a}
$$


Corollary 5.3. Suppose that the survival rate is given by Type I or by Type II. Then the basic reproduction number is approximately given by

$$
R_{0} \approx \frac{1}{A b_{0}}
$$

Proof. If we use Type II survival function $\ell(a)=e^{-\mu a}$, then

$$
b_{0}=r_{0}+\mu, \quad x^{*}=\frac{b_{0}}{\lambda^{*}+\mu+r_{0}}, \quad A=\frac{1}{\lambda^{*}+\mu+r_{0}} .
$$

Therefore from (5.19) we have

$$
R_{0} \approx \frac{1}{x^{*}}=\frac{1}{A b_{0}}
$$

Next if we use Type I survival rate, that is, there exists a maximal age $\omega$ such that $\ell(a)=1$ for $a \in[0, \omega)$ and $\ell(a)=0$ for $a \geq \omega$, then we have

$$
b_{0}=\frac{r_{0}}{1-e^{-r_{0} \omega}}, \quad x^{*}=\frac{b_{0}\left(1-e^{-\left(r_{0}+\lambda^{*}\right) \omega}\right)}{r_{0}+\lambda^{*}}, \quad A=\frac{1}{\lambda^{*}+r_{0}}-\frac{\omega e^{-\left(r_{0}+\lambda^{*}\right) \omega}}{1-e^{-\left(r_{0}+\lambda^{*}\right) \omega}} .
$$

Thus it follows that

$$
R_{0}=\frac{1}{b_{0}} \frac{r_{0}+\lambda^{*}}{1-e^{-\left(r_{0}+\lambda^{*}\right) \omega}}=\frac{1+\epsilon}{b_{0} A}
$$

where

$$
\epsilon:=\frac{e^{-\left(r_{0}+\lambda^{*}\right) \omega}}{1-e^{-\left(r_{0}+\lambda^{*}\right) \omega}}-\frac{\left(r_{0}+\lambda^{*}\right) \omega e^{-\left(r_{0}+\lambda^{*}\right) \omega}}{\left(1-e^{-\left(r_{0}+\lambda^{*}\right) \omega}\right)^{2}} .
$$

For common childhood diseases, $\epsilon$ is very small, so we can again conclude that the formula

$$
R_{0} \approx \frac{1}{A b_{0}}
$$

holds for the Type I survival rate.

The estimate (5.23) has been derived for growing populations with the pseudo massaction type (scale-dependent) FOI [10] by May and Anderson [40].

Next let us examine the effect of the population growth rate on the basic reproduction number:

Proposition 5.4. If the transmission rate $\beta$ is constant, the basic reproduction number $R_{0}$ is a decreasing function of the intrinsic rate of natural increase $r_{0}$.

Proof. To see this statement, it is sufficient to show that $x^{*}$ is an increasing function of $r_{0}$. By basic calculation of derivatives, it is easy to see that

$$
\frac{d x^{*}}{d r_{0}}=x^{*}\left(A_{1}-A_{2}\right),
$$


where

$$
A_{1}:=\int_{0}^{\infty} a \psi(a) d a, \quad A_{2}:=\frac{\int_{0}^{\infty} a s^{*}(a) e^{-r_{0} a} \ell(a) d a}{\int_{0}^{\infty} s^{*}(a) e^{-r_{0} a} \ell(a) d a} .
$$

Then $A_{1}$ denotes the average age of the host stable population and $A_{2}$ is the average age of the susceptible population, hence $A_{1}-A_{2}>0$. Then we know that $x^{*}$ is monotone increasing with respect to $r_{0}$.

Therefore, against our intuition, developing countries with higher intrinsic growth rate may have smaller $R_{0}$ in comparison with developed countries with low intrinsic growth rate. This fact has been suggested by McLean [41] and examined by Inaba [28] for the SIR epidemic in a stable population.

From (5.19) and (5.29), we obtain

$$
\frac{d R_{0}}{d r_{0}} \approx-\frac{1}{x^{*}} \frac{d x^{*}}{d r_{0}} R_{0}=-R_{0}\left(A_{1}-A_{2}\right)
$$

Then if $\Delta r_{0}$ is a perturbation in $r_{0}$, the relative change of $R_{0}$ is given by

$$
\frac{\Delta R_{0}}{R_{0}} \approx-\left(A_{1}-A_{2}\right) \Delta r_{0}
$$

For example, if $\Delta r_{0}=-0.01$ and $A_{1}-A_{2}=30, R_{0}$ will show a 30 percent increase, which shows that developed countries with below replacement fertility and aged populations are likely to be invaded by infectious diseases if their $\beta$ could be assumed to be age-independent.

\section{Vaccination}

Let $\eta(a)$ be the age-dependent force of vaccination and we assume that the host population has already attained a partially immunized stationary state by the vaccination policy described by $\eta$. Then the susceptible population's (normalized) density in the partially immunized disease-free steady state is given as

$$
s_{\eta}(a)=H(a),
$$

where $H$ is the survival rate corresponding to the force of vaccination $\eta$ :

$$
H(a):=\exp \left(-\int_{0}^{a} \eta(\zeta) d \zeta\right)
$$

Then, if it exists, the endemic steady state with vaccination is given by

$$
s_{\eta}^{*}(a)=s_{\eta}(a) \Lambda_{\eta}^{*}(a)=\Lambda_{\eta}^{*}(a) H(a),
$$

where $\Lambda_{\eta}^{*}$ is the survival rate associated with the force of infection $\lambda_{\eta}^{*}$ at the endemic steady state with vaccination given by

$$
\Lambda_{\eta}^{*}(a):=\exp \left(-\int_{0}^{a} \lambda_{\eta}^{*}(\zeta) d \zeta\right)
$$


where $\lambda_{\eta}^{*}$ is the positive solution of a nonlinear integral equation:

$$
\lambda_{\eta}^{*}(a)=\int_{0}^{\infty} \int_{0}^{\infty} \beta(a, \zeta+\tau) \psi(\zeta+\tau) f(\tau) \Gamma(\tau) d \tau \lambda_{\eta}^{*}(\zeta) s_{\eta}^{*}(\zeta) d \zeta
$$

Let $K_{\eta}$ be the effective next generation operator (ENGO) with vaccination given by

$$
K_{\eta}=s_{\eta} K
$$

Now we know that there exists at least one endemic steady state with vaccination if and only if $R_{\eta}:=r\left(K_{\eta}\right)>1$, where $R_{\eta}$ is the effective reproduction number under the vaccination policy $\eta$.

The newly infected population density at ESS with vaccination, denoted by $\xi_{\eta}^{*}$, satisfies the renewal equation

$$
\xi_{\eta}^{*}(a)=\lambda_{\eta}^{*}(a) s_{\eta}^{*}(a) \psi(a)=\left(T_{\eta} \xi_{\eta}^{*}\right)(a)
$$

where $T_{\eta}$ denotes the ENGO at ESS with vaccination given by

$$
T_{\eta}=\left[\frac{s_{\eta}^{*}}{s_{\eta}}\right] K_{\eta}=\Lambda_{\eta}^{*} K_{\eta}=s_{\eta}^{*} K=\left[\frac{s_{\eta}^{*}}{s^{*}}\right] T .
$$

Let $F_{\eta}$ be the adjoint eigenfunctional of $K_{\eta}$ associated with its spectral radius (Frobenius eigenvalue) $R_{\eta}$. Then we can observe that

$$
<K_{\eta}^{*} F_{\eta}, \xi_{\eta}^{*}>=R_{\eta}<F_{\eta}, \xi_{\eta}^{*}>=<F_{\eta}, K_{\eta} \xi_{\eta}^{*}>
$$

Hence we have the estimation formula as

$$
R_{\eta}=\frac{<F_{\eta}, K_{\eta} \xi_{\eta}^{*}>}{<F_{\eta}, \xi_{\eta}^{*}>} .
$$

Here we again adopt the proportionate mixing assumption. Then $K_{\eta}$ becomes a onedimensional operator:

$$
\left(K_{\eta} \phi\right)(a)=s_{\eta}(a)(K \phi)(a)=H(a)<F_{0}, \phi>\beta_{1}(a) \psi(a),
$$

Then the Frobenius eigenvector of $K_{\eta}$ is $H \beta_{1} \psi$ and it follows that

$$
R_{\eta}=r\left(K_{\eta}\right)=<F_{0}, H \beta_{1} \psi>
$$

Lemma 6.1. Under the proportionate mixing assumption, the dual eigenfunctional $F_{\eta}$ of $K_{\eta}$ associated with $R_{\eta}$ is proportional to $F_{0}$.

Proof. For any $\phi \in L^{1}$, it follows from (6.9) that

$$
\begin{aligned}
<F_{\eta}, K_{\eta} \phi> & =<K_{\eta}^{*} F_{\eta}, \phi>=R_{\eta}<F_{\eta}, \phi> \\
& =<F_{0}, \phi><F_{\eta}, H \beta_{1} \psi>,
\end{aligned}
$$


which shows that

$$
R_{\eta} F_{\eta}=<F_{\eta}, H \beta_{1} \psi>F_{0}
$$

Then $F_{\eta}$ is proportional to $F_{0}$.

On the other hand, it follows from (6.3)-(6.5) that

$$
\psi(a) \lambda_{\eta}^{*}(a)=\frac{1}{s_{\eta}(a)}\left(K_{\eta} \xi_{\eta}^{*}\right)(a)=<F_{0}, \xi_{\eta}^{*}>\beta_{1}(a) \psi(a) .
$$

Then from (6.2) and (6.5) we obtain

$$
\xi_{\eta}^{*}(a)=\lambda_{\eta}^{*}(a) s_{\eta}^{*}(a) \psi(a)=<F_{0}, \xi_{\eta}^{*}>\beta_{1}(a) H(a) \Lambda_{\eta}^{*}(a) \psi(a) .
$$

Applying (6.9) and (6.12) to (6.8) and using Lemma 6.1, we arrive at the estimation formula:

$$
R_{\eta}=\frac{<F_{0}, H \beta_{1} \psi>}{<F_{0}, H \beta_{1} \Lambda_{\eta}^{*} \psi>} .
$$

Note that it follows from (6.6) and (6.9) that

$$
\left(T_{\eta} \phi\right)(a)=<F_{0}, \phi>H(a) \Lambda_{\eta}^{*}(a) \beta_{1}(a) \psi(a) .
$$

From the stationarity condition, we have

$$
r\left(T_{\eta}\right)=<F_{0}, H \Lambda_{\eta}^{*} \beta_{1} \psi>=1 .
$$

Then expressions (6.10) and (6.13) are equivalent to each other, but the expression (6.13) is again more advantageous to eliminate unknown scale factors commonly included in the numerator and the denominator.

If we again assume that $\beta_{1}$ and $\beta_{2}$ are proportional, then they are necessarily proportional to $\lambda_{\eta}^{*}$ and we obtain the following estimate:

$$
\begin{aligned}
R_{\eta} & =\frac{<F_{0}, H \lambda_{\eta}^{*} \psi>}{<F_{0}, H \lambda_{\eta}^{*} \Lambda_{\eta}^{*} \psi>} \\
& =\frac{\int_{0}^{\infty} \int_{0}^{\infty} \lambda_{\eta}^{*}(\tau+\zeta) \psi(\tau+\zeta) f(\tau) \Gamma(\tau) d \tau \lambda_{\eta}^{*}(\zeta) H(\zeta) d \zeta}{\int_{0}^{\infty} \int_{0}^{\infty} \lambda_{\eta}^{*}(\tau+\zeta) \psi(\tau+\zeta) f(\tau) \Gamma(\tau) d \tau \lambda_{\eta}^{*}(\zeta) s_{\eta}^{*}(\zeta) d \zeta}
\end{aligned}
$$

Moreover if we assume that the infectious period is short enough, we obtain

$$
R_{\eta} \approx \frac{\int_{0}^{\infty} \lambda_{\eta}^{* 2}(\zeta) H(\zeta) \psi(\zeta) d \zeta}{\int_{0}^{\infty} \lambda_{\eta}^{* 2}(\zeta) s_{\eta}^{*}(\zeta) \psi(\zeta) d \zeta}
$$

Note that if we can observe the incidence of infection at the endemic steady state $\xi_{\eta}^{*}$ and the vaccination survival rate $H$, we can estimate the FOI $\lambda_{\eta}^{*}$ from the relation $\xi_{\eta}^{*}=\lambda_{\eta}^{*} \Lambda_{\eta}^{*} H \psi$ as

$$
\lambda_{\eta}^{*}(a)=\frac{\xi_{\eta}^{*}(a)}{\psi(a) H(a)\left(1-\int_{0}^{a} \frac{\xi_{\eta}^{*}(\sigma)}{\psi(\sigma) H(\sigma)} d \sigma\right)} .
$$


Then we can use (6.17) to estimate $R_{\eta}$.

On the other hand, if the FOI without vaccination is known, we can replace the denominator of (6.13) by the relation (5.10) (both are equal to unity) to arrive at the estimate:

$$
R_{\eta}=\frac{<F_{0}, H \beta_{1} \psi>}{<F_{0}, \beta_{1} s^{*} \psi>}
$$

By using the same argument as above, if $\beta_{1}$ and $\beta_{2}$ are proportional, then they are proportional to $\lambda^{*}$, we have

$$
\begin{aligned}
R_{\eta}= & \frac{<F_{0}, H \lambda^{*} \psi>}{<F_{0}, \lambda^{*} s^{*} \psi>} \\
= & \frac{\int_{0}^{\infty} \int_{0}^{\infty} \lambda^{*}(\tau+\zeta) \psi(\tau+\zeta) f(\tau) \Gamma(\tau) d \tau \lambda^{*}(\zeta) H(\zeta) d \zeta}{\int_{0}^{\infty} \int_{0}^{\infty} \lambda^{*}(\tau+\zeta) \psi(\tau+\zeta) f(\tau) \Gamma(\tau) d \tau \lambda^{*}(\zeta) s^{*}(\zeta) d \zeta}
\end{aligned}
$$

Again if we assume that the infectious period is short enough, we obtain

$$
R_{\eta} \approx \frac{\int_{0}^{\infty} \lambda^{* 2}(\zeta) H(\zeta) \psi(\zeta) d \zeta}{\int_{0}^{\infty} \lambda^{* 2}(\zeta) s^{*}(\zeta) \psi(\zeta) d \zeta} .
$$

The FOI $\lambda^{*}$ can be estimated from the incidence without vaccination $\xi^{*}(a)$ by using (5.14), then we can use (6.21) to estimate $R_{\eta}$.

As an example, suppose that the force of vaccination is given by

$$
\eta(a)=-\log (1-p) \delta\left(a-a_{0}\right), \quad p \in(0,1),
$$

where $\delta$ denotes the delta function. Then it follows that

$$
H(a)=\left\{\begin{array}{ll}
1 & 0 \leq a<a_{0} \\
1-p & a_{0}<a
\end{array},\right.
$$

which implies that susceptible individuals are vaccinated only once at age $a_{0}$ and $p$ is its vaccination coverage. Then it follows from (6.21) that

$$
\begin{aligned}
R_{\eta} \approx & \frac{\int_{0}^{a_{0}} \lambda^{* 2}(\zeta) \psi(\zeta) d \zeta+(1-p) \int_{a_{0}}^{\infty} \lambda^{* 2}(\zeta) \psi(\zeta) d \zeta}{\int_{0}^{\infty} \lambda^{* 2}(\zeta) s^{*}(\zeta) \psi(\zeta) d \zeta} \\
& =R_{0}\left(1-p \frac{\int_{a_{0}}^{\infty} \lambda^{* 2}(\zeta) \psi(\zeta) d \zeta}{\int_{0}^{\infty} \lambda^{* 2}(\zeta) \psi(\zeta) d \zeta}\right)
\end{aligned}
$$

Therefore the subcritical condition $R_{\eta}<1$ is realized if and only if

$$
p>\left(1-\frac{1}{R_{0}}\right) \frac{\int_{0}^{\infty} \lambda^{* 2}(\zeta) \psi(\zeta) d \zeta}{\int_{a_{0}}^{\infty} \lambda^{* 2}(\zeta) \psi(\zeta) d \zeta}
$$


In particular, if we assume that $\lambda^{*}$ is constant, the condition (6.25) is reduced to the condition

$$
p>\left(1-\frac{1}{R_{0}}\right) \frac{1}{\int_{a_{0}}^{\infty} \psi(\zeta) d \zeta},
$$

where $\int_{a_{0}}^{\infty} \psi(\zeta) d \zeta$ is the proportion of population whose age is above $a_{0}$ in the stable population. Therefore if

$$
R_{0} \geq \frac{1}{\int_{0}^{a_{0}} \psi(\zeta) d \zeta}
$$

then there is no $p \in[0,1]$ that satisfies $(6.26)$, so the disease can not be eradicated by the one time mass vaccination at age $a_{0}$. Roughly speaking, in order to control an infectious disease by one time mass vaccination, the proportion of population below vaccination age in a host stable population must be less than $1 / R_{0}$.

If $\lambda^{*}$ is constant, it follows from (6.24) and (5.19) that

$$
R_{\eta} \approx \frac{1}{x^{*}}\left(1-p \int_{a_{0}}^{\infty} \psi(\zeta) d \zeta\right)
$$

Then it is easy to see that

$$
\frac{d R_{\eta}}{d r_{0}} \approx \frac{1}{x^{*}}\left[-(1-p)\left(A_{1}-A_{2}\right)+p\left(A_{2}-A_{3}\right) \int_{0}^{a_{0}} \psi(\zeta) d \zeta\right]
$$

and $A_{3}$ is the average age of population whose age is less than $a_{0}$ in the stable population:

$$
A_{3}:=\frac{\int_{0}^{a_{0}} \zeta \psi(\zeta) d \zeta}{\int_{0}^{a_{0}} \psi(\zeta) d \zeta} .
$$

Therefore, in contrast with $R_{0}$, the effective reproduction number with vaccination $R_{\eta}$ is not necessarily a decreasing function of the intrinsic growth rate $r_{0}$.

\section{Numerical Examples}

Here we numerically illustrate the impact of population growth on threshold condition of an infectious disease. We firstly examine the basic reproduction number, $R_{0}$, and the average age at infection in a population with various population growth rates. Subsequently, as implications for mass vaccination strategy, critical condition of eradication and age of mass vaccination are discussed for both growing and decreasing populations.

\subsection{Estimation of $R_{0}$ in Growing and Decreasing Populations}

In the following, $R_{0}$ is estimated employing simple assumptions for the force of infection and host population dynamics. The force of infection, $\lambda$, can be reasonably estimated using 


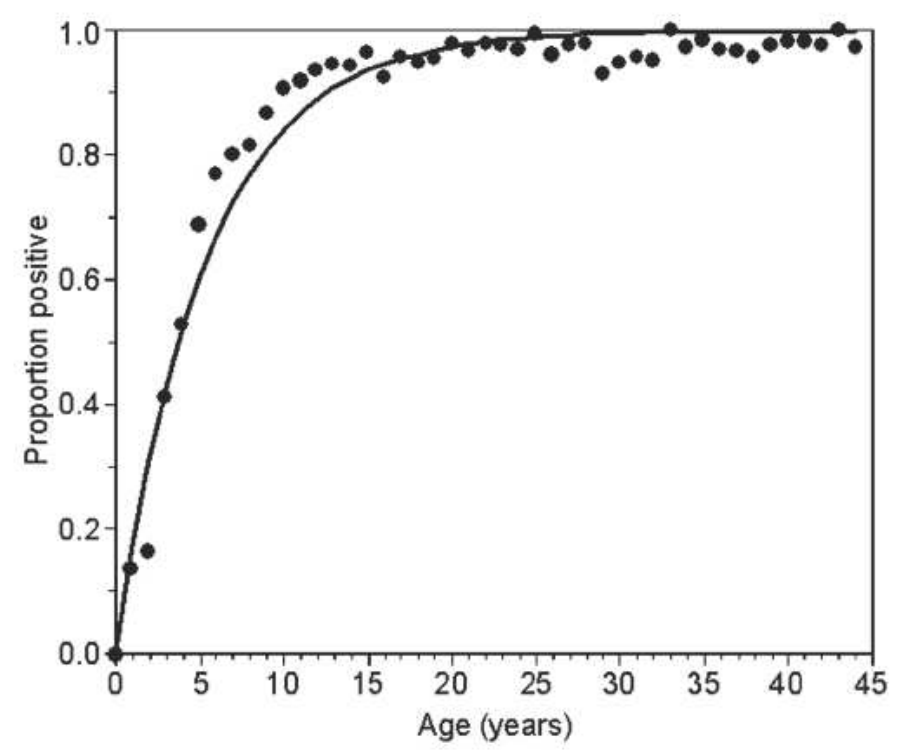

Figure 1: Distribution of the age at infection of mumps in the United Kingdom, 1986-7. Shows the observed and predicted proportions of those exposed to mumps. Each dot shows the observed data and the solid line the predicted data. The estimate of the force of infection, $\lambda$, is 0.184 per year. Data source: [17].

age-specific cumulative proportion of infection [17]. For simplicity, we assume that $\lambda$ is ageindependent. Assuming further that all individuals are susceptible to a disease at age 0 , the cumulative proportion of infection, $G(a)$, is given by

$$
G(a)=1-\exp (-\lambda a)
$$

Suppose that there are $N_{a}$ individuals who had been seropositive until age $a$ and $M_{a}$ who had not, the likelihood function to estimate $\lambda$ is

$$
L(\lambda)=\prod_{a} G_{a}^{N_{a}}\left(1-G_{a}\right)^{M_{a}}
$$

where $G_{a}$ is discrete approximation of (7.1), yielding the proportion seropositive in the discretized age group $a$ [23]. Figure 1 shows the age-specific proportions of those experiencing mumps infection until age $a$ in the United Kingdom from 1986-7 [17]. Applying the above described simple model to the data, maximum likelihood estimate of $\lambda$ is 0.184 (95 percent confidence interval (CI): 0.178, 0.190) per year.

Now, we consider a population following Type I survivorship with the average life expectancy at birth, $\omega$, being 70 years. In a stationary population with zero-population growth 
rate (i.e. $\left.r_{0}=0\right)$, an estimator of $R_{0}$ is reduced from (5.27) to

$$
R_{0}=\frac{\lambda \omega}{1-\exp (-\lambda \omega)}
$$

which has been relatively well-known since a pioneering note of Dietz [13]. Using this, $R_{0}$ is reasonably estimated as 12.9 (95 percent CI: 12.4, 13.3). Assuming that the mumps transmission has achieved stationary state in a hypothetical population, the proportion of those who experience infection until age $\omega=70$ years is 99.99997 percent (95 percent CI: 99.99996, 99.99998) from (7.1). Applying this to (5.15), exactly the same estimate of $R_{0}$ as given by (7.3) is obtained. It should be noted that we have to measure the final size of a cohort very precisely to estimate $R_{0}$ using (5.15). That is, for a disease with which almost all individuals experience infection before their deaths, (5.15) may have limited practical utility (compared with (7.3)). Rather, (5.15) is intended to enhance theoretical understanding of an age-specific transmission in a stationary population assuming Type I survivorship.

Let us then consider growing and decreasing populations. Sensitivities of $R_{0}$ and average age at infection are examined in relation to the host population growth rate, $r_{0}$. In addition to $\lambda=0.20$ per year, which roughly corresponds to estimate in the above mumps case, we conduct sensitivity analysis using small and extremely large $\lambda$, i.e., 0.05 and 0.50 per year. Two different survivorship functions, Type I and II, are assumed for the host population dynamics; in both assumptions, the average life expectancy at birth is assumed to be 70 years. Then, the equations (5.24)-(5.26) can be reasonably applied to the data with the above described assumptions.

Figure 2A examines sensitivity of $R_{0}$ to different values of $r_{0}$. For both survivorship assumptions, a clear tendency is seen. That is, the smaller $r_{0}$ is, the greater $R_{0}$ would be. This is particularly highlighted for Type II survivorship assumption and for large $\lambda$, especially when $r_{0}$ is negative (i.e. decreasing population). Although in a stationary population estimates of $R_{0}$ assuming Type II survivorship have been known to be higher than those assuming Type I (given the same $\lambda$ ), this is not the case for rapidly growing population. Figure 2B illustrates sensitivity of the average age at infection to various $r_{0}$. Average age at infection is not greatly influenced by $r_{0}$ for large $\lambda$. However, for small $\lambda$, the average age at infection is lowered as $r_{0}$ increases. In the given range of $r_{0}$, qualitative pattern of the average age at infection is not considerably different between Type I and II assumptions. In practical term, Figure 2 implies that $R_{0}$ in a decreasing population is greater than that of growing population, for the same disease. This relationship highlights that $R_{0}$ is most likely higher in industrialized countries than in developing countries [41], given the same force of infection. In addition, the above exercise suggests an important implication for statistical estimation of $R_{0}$; although previous modeling frameworks tended to estimate key parameters of transmission often assuming a stationary population mostly without any justification, it is critically important to account for the host population growth rate, especially in rapidly growing and decreasing populations [42]. 

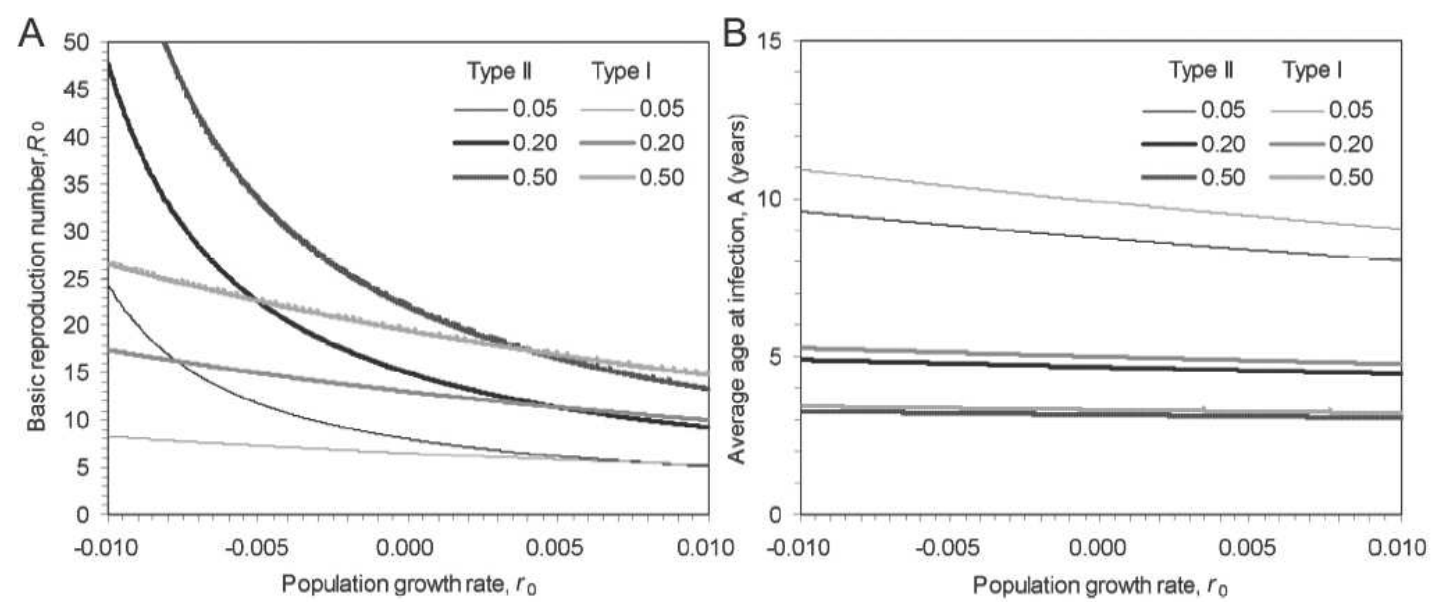

Figure 2: Sensitivity of the basic reproduction number and average age at infection to different values of population growth rate. [A]. The basic reproduction number, $R_{0}$ vs population growth rate, $r_{0}$, with different forces of infection $\lambda$. [B]. Average age at infection, $A$ vs population growth rate, $r_{0}$ with different $\lambda$. Assumed values for $\lambda$ were 0.05 (thin), 0.20 (thick) and 0.50 (thick and shaded) per year. Two different survivorship functions, Types I (grey) and II (black), were assumed for the host population dynamics; for both assumptions, the average life expectancy at birth was assumed to be 70 years. See equations from (5.24)-(5.26) for details of the model.

\subsection{Eradication Thresholds in Growing and Decreasing Popula- tions}

We then investigate the sensitivity of eradication threshold to different values of $r_{0}$. Assuming Type II survivorship with the constant force of death, $\mu=1 / 70$ per year, the normalized stable age distribution, $\psi(a)$, in $(2.8)$ is

$$
\psi(a)=b_{0} \exp \left(-\left(r_{0}+\mu\right) a\right) .
$$

Thus, the condition (6.26) is rewritten as

$$
\begin{aligned}
p & >\left(1-\frac{1}{R_{0}}\right) \frac{1}{\int_{a_{0}}^{\infty} b_{0} \exp \left(-\left(r_{0}+\mu\right) \zeta\right) d \zeta} \\
& =\left(1-\frac{1}{R_{0}}\right) \frac{1}{\exp \left(-\left(r_{0}+\mu\right) a_{0}\right)} .
\end{aligned}
$$

As an example, we consider the case when mass vaccination against measles is performed, only once in life, exactly and immediately at the age of 3 (i.e. $a_{0}=3$ years). Following previous estimates, $R_{0}$ is assumed to range from 5-15 ([16], [20], [45], [49], [53], [57]). 


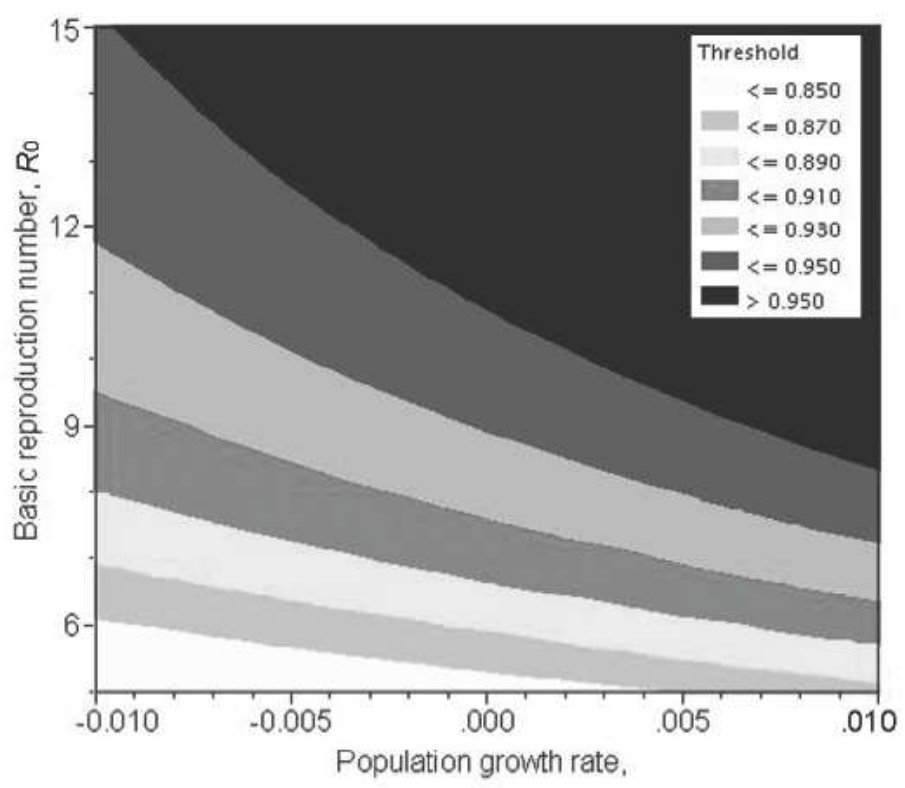

Figure 3: Eradication threshold of a disease in a population with various growth rates. Sensitivity of the threshold condition for eradication to different values of the basic reproduction number, $R_{0}$ and population growth rates, $r_{0}$ is examined. The mass vaccination is assumed to be completed exactly and immediately at the age of 3 years $\left(=a_{0}\right)$. Type II survivorship is assumed for the host population dynamics with the constant force of death, $\mu=1 / 70$ per year. See equations (2.8) and (6.26) for details of the model.

Figure 3 shows the eradication threshold with various $R_{0}$ and $r_{0}$. Clearly reflecting (7.5), we need to vaccinate considerably high proportion of susceptible individuals for large $r_{0}$ as well as $R_{0}$ in a growing population. The eradication threshold is highly sensitive to $r_{0}$ in decreasing population, lowering critical coverage of vaccination even for large $R_{0}$. These findings suggest that the disease control by mass vaccination is more difficult in growing population than in decreasing population, given the same $R_{0}$.

Figure 4 compares the critical coverage of vaccination between growing (panel A) and decreasing populations (B) by different ages to complete mass vaccination. For growing and decreasing populations, the population growth rates, $r_{0}$, are assumed to be 0.01 and -0.01 per year, respectively. Other conditions (e.g. Type II survivorship with $\mu=1 / 70$ per year) are the same as analyzed in Figure 3. In the growing population (Figure 4A), threshold is highly sensitive to the age to complete vaccination, implying that eradication needs to achieve mass vaccination campaign at the very small age in addition to a need for high coverage. On the other hand, Figure 4B shows that the threshold becomes far less sensitive to the age of vaccination in decreasing population. That is, a slight delay in the age of mass vaccination would not significantly vary the critical coverage in the decreasing population. 

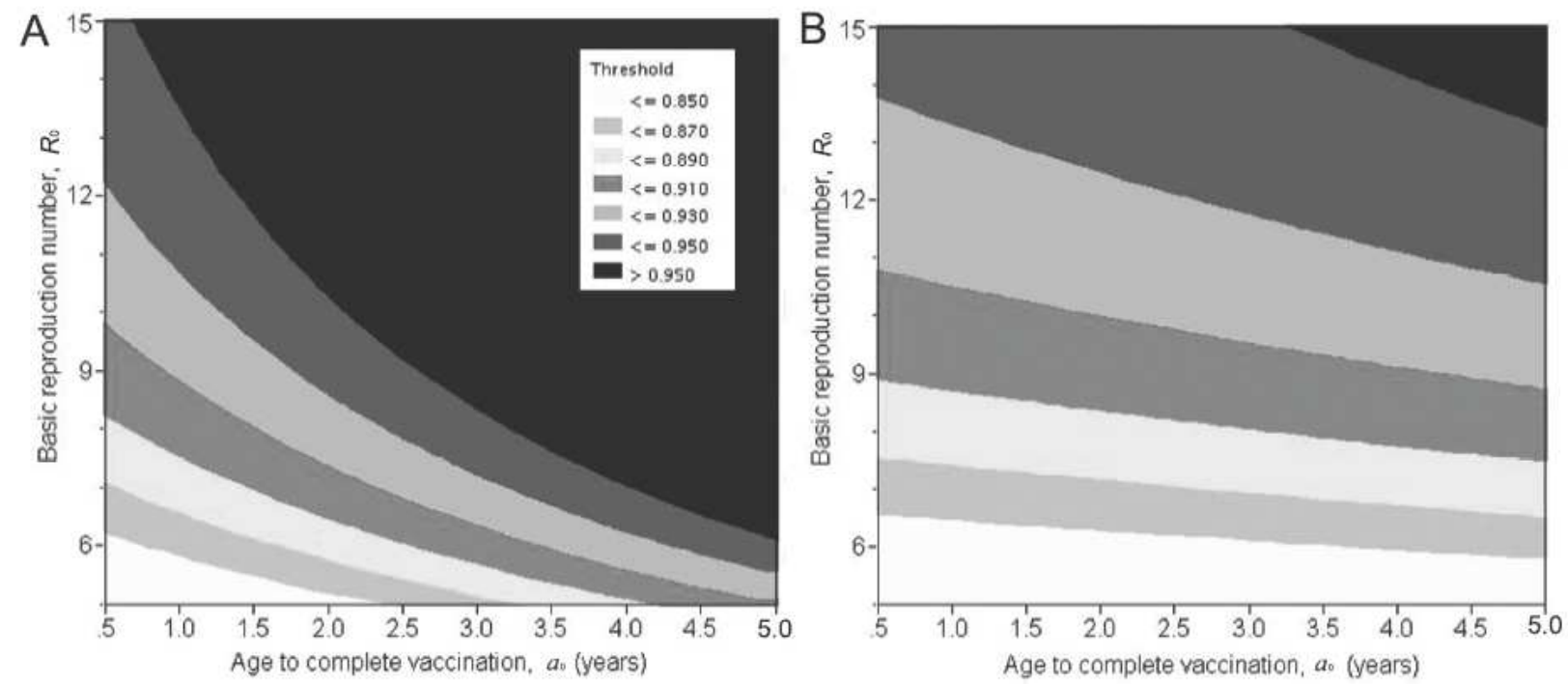

Figure 4: Comparison of the eradication thresholds of a disease between growing and decreasing populations with different ages to complete vaccination. Sensitivity of the threshold condition for eradication to different values of the basic reproduction number, $R_{0}$ and age to complete vaccination, $a_{0}$ is examined. Whereas the panel $\mathbf{A}$ shows the thresholds for increasing population with population growth rate, $r_{0}=0.01$ per year, panel $\mathbf{B}$ shows those for decreasing population with $r_{0}=-0.01$ per year. Type II survivorship is assumed for the host population dynamics with the constant force of death, $\mu=1 / 70$ per year. See equations (2.8) and (6.26) for details of the model.

In practical term, mass vaccination needs to be done at an early age with high coverage in rapidly growing population (e.g. developing country), though it should be noted that our exercise has not taken into account other important factors such as maternal antibody [34], $[43])$.

\section{Discussion}

In this paper, based on the age-duration-structured SIR epidemic system, we have established the basic reproduction number, the endemic and the eradication thresholds in a stable (host) population, and examined possible impact of population growth on those epidemic parameters. We have also shown that threshold conditions are well formulated by the (effective) next generation operators.

The basic reproduction number $R_{0}$ was analytically derived under different assumptions of the host population dynamics and the force of infection. In particular, extending previous findings of Dietz and Schenzle [14] and Farrington, Kanaan and Gay [17], various estimators 
of $R_{0}$ were derived, explicitly taking into account the host population growth rate. As the special cases, it was shown that the estimator of $R_{0}$ is approximately reduced to $1 / A b_{0}$ for both Type I and II populations with the age-independent force of infection, where $A$ and $b_{0}$ are the average age at infection and the crude birth rate of the population.

Using analytical results in Sections 5 and 6, numerical exercises were performed. Through the estimation of $R_{0}$ assuming an age-independent force of infection, it was demonstrated that $R_{0}$ increases as the population growth rate $r_{0}$ decreases. This was particularly the case for the population following Type II survivorship. In addition, although previous analyses have shown that, given the same force of infection, estimate of $R_{0}$ assuming Type II survivorship would be greater than that assuming Type I in a stationary population ([3], [13]), we observed that this would not be the case for rapidly growing population. In practical term, these results indicate that $R_{0}$ in the developing countries would be smaller than that in developed countries, which is consistent with previous suggestions ([41], [33], [52], [28]).

Moreover, we investigated the threshold condition to eradicate a disease using our analytical results, with particular emphasis on vaccination. Given the same $R_{0}$, critical coverage of vaccination in a growing population would be higher than that of decreasing population. We further used the analytical results to investigate the age to vaccinate susceptible children in a mass vaccination program. Whereas the critical coverage of vaccination was sensitive to the age of vaccination in growing population, the threshold was less sensitive to the age of vaccination in decreasing population. The finding highlights a difficulty in eradicating a disease by means of incomplete mass vaccination in developing countries, suggesting a need for high coverage of vaccination performed at an early age. Although it is important to take into account other intrinsic factors to appropriately discuss the mass vaccination strategy (e.g. maternal antibody [34], [43]), key features of the intervention threshold was highlighted in relation to our analytical solutions under simple assumptions.

To date, there have been various studies on epidemics in a population with varying size. Following a pioneering study by May and Anderson [40], this issue has been discussed mainly based on a system governed by ordinary differential equations ([41], [4], [5], [6], [7], [47]). Mathematical extensions have been made particularly with respect to the assumption of the loss of acquired immunity which was incorporated in the similar system ([9], [19], [35]). In practice, only a few quantitative studies investigated practical implications of varying population size using the observed data, e.g., in ageing and decreasing population in Italy ([36], [58]). In addition to a limited number of rigorous studies exploring the similar age-structured system with varying population size ([32], [33], [52]), the present study has shown analytical solutions of the age-duration-structured SIR system, using the next generation arguments and explicitly incorporating varying age-distribution of the host population. Further, the present study is the first to suggest several estimators of $R_{0}$ and threshold of vaccination, which were motivated by a need to apply the concept to observed data. We believe our estimators in Sections 4 and 5 will be useful to statistically analyze the data in both growing and decreasing populations.

Several questions remain for future studies. For example, when we have to deal with the loss of immunity (e.g. SIRS model), it has not been known if any conditions of endemic 
steady state can be derived using the age-structured system. If we see the SIRS system as a special case of the SIR model with variable forces of reinfection, the problem would be more tractable [27]. Moreover, epidemic with varying population size would be particularly of interest when an infectious disease is highly fatal and influences the host demographic structure ([5], [50]); the basic idea of such an issue has been elaborated with regard to HIV/AIDS in Sub-Saharan African countries [2]. Since statistical estimation and practical utility are enhanced by offering estimators derived from analytical solutions of an explicitly structured system, we believe our study contributes to future applications and extensions.

\section{References}

[1] R. M. Anderson, R. M. May. Directly transmitted infectious diseases: control by vaccination. Science, 215 (1982), 1053-1060.

[2] R. M. Anderson, R. M. May, A. R. McLean. Possible demographic consequences of AIDS in developing countries. Nature. 332 (1988), 228-234.

[3] R. M. Anderson, R. M. May. Infectious Diseases of Humans: Dynamics and Control, Oxford UP, Oxford, 1991.

[4] F. Brauer. Epidemic models in populations of varying size. In: Mathematical approaches to resource management and epidemiology, C. Castillo-Chavez, S.A. Levin and C.A. Shoemaker (eds.), Springer-Verlag, New York, 1989, 109-123.

[5] S. Busenberg, K. P. Hadeler. Demography and epidemics. Math. Biosci. 101 (1990), 41-62.

[6] S. Busenberg, P. van den Driessche. Analysis of a disease transmission model in a population with varying size. J. Math. Biol. 28 (1990), 257-270.

[7] S. Busenberg, P. van den Driessche. Nonexistence of periodic solutions for a class of epidemiological models. In: Biology, Epidemiology, and Ecology, S. Busenberg and M. Martelli (eds.), Lect. Notes Biomath 92, Springer, Berlin, 1991, 70-79.

[8] S. Busenberg, K. Cooke. Vertically transmitted diseases: models and dynamics. Biomathematics 23, Springer, Berlin, 1993.

[9] W. R. Derrick, P. van den Driessche. A disease transmission model in a nonconstant population. J. Math. Biol., 31 (1993), 495-512.

[10] M. C. M. de Jong, O. Diekmann, H. Heesterbeek. How does transmission of infection depend on population size? In: Epidemic Models: Their Structure and Relation to Data, D. Mollison (ed.), Cambridge U. P., Cambridge, 1995, 84-94. 
[11] O. Diekmann, J. A. P. Heesterbeek, J. A. J. Metz. On the definition and the computation of the basic reproduction ratio $R_{0}$ in models for infectious diseases in heterogeneous populations. J. Math. Biol., 28 (1990), 365-382.

[12] O. Diekmann, J. A. P. Heesterbeek. Mathematical epidemiology of infectious diseases: model building, analysis and interpretation. John Wiley and Sons, Chichester, 2000.

[13] K. Dietz. Transmission and control of arbovirus diseases. In: Proceedings of SIMS Conference on Epidemiology, D. Ludwig and K. L. Cooke (eds.), SIAM, Philadelphia, $1975,104-121$.

[14] K. Dietz, D. Schenzle. Proportionate mixing models for age-dependent infection transmission. J. Math. Biol. 22 (1985), 117-120.

[15] W. J. Edmunds, C. J. O'Callaghan, D. J. Nokes. Who mixes with whom? A method to determine the contact patterns of adults that may lead to the spread of airborne infections. Proc. R. Soc. Lond. Ser. B, 264 (1997), 949-957.

[16] W. J. Edmunds, N. J. Gay, M. Kretzschmar, R. G. Pebody, H. Wachmann, ESEN Project European Sero-epidemiology Network. The pre-vaccination epidemiology of measles, mumps and rubella in Europe: implications for modelling studies. Epidemiol. Infect., 125 (2000), 635-650.

[17] C. P. Farrington, M. N. Kanaan, N. J. Gay. Estimation of the basic reproduction number for infectious diseases from age-stratified serological survey data. Appl. Statist., 50, Part 3 (2001), 251-292.

[18] C. P. Farrington, H. J. Whitaker. Contact surface models for infectious diseases: estimation from serologic survey data. J. Am. Stat. Assoc., 100 (2005), 370-379.

[19] L. Q. Gao, H. W. Hethecote. Disease transmission models with density-dependent demographics. J. Math. Biol., 30 (1992), 717-731.

[20] R. F. Grais, M. J. Ferrari, C. Dubray, O. N. Bjornstad, B. T. Grenfell, A. Djibo, F. Fermon, P.J. Guerin. Estimating transmission intensity for a measles epidemic in Niamey, Niger: lessons for intervention. Trans. R. Soc. Trop. Med. Hyg., 100 (2006), 867-873.

[21] D. Greenhalgh, K. Dietz. Some bounds on estimates for reproductive ratios derived from the age-specific force of infection. Math. Biosci., 124 (1994), 9-57.

[22] B. T. Grenfell, R. M. Anderson. The estimation of age-related rates of infection from case notifications and serological data. J. Hyg (Lond)., 95 (1985), 419-436.

[23] D. A. Griffiths. A catalytic model of infection for measles. Appl. Stat., 23 (1974), 330-339. 
[24] H. J. A. M. Heijmans. The dynamical behaviour of the age-size-distribution of a cell population. In: The Dynamics of Physiologically Structured Populations, J. A. J. Metz and O. Diekmann (eds.), Lect. Notes Biomath. 68, Springer-Verlag, Berlin, 1986, 185-202.

[25] M. Iannelli. Mathematical theory of age-structured population dynamics. Giardini Editori e Stampatori in Pisa, 1995.

[26] H. Inaba. Threshold and stability results for an age-structured epidemic model. J. Math. Biol., 28 (1990), 411-434.

[27] H. Inaba. Kermack and McKendrick revisited: The variable susceptibility model for infectious diseases. Japan J. Indust. Appl. Math., 18 (2001), No. 2, 273-292.

[28] H. Inaba. Mathematical analysis of an age-structured SIR epidemic model with vertical transmission. Disc. Cont. Dyn. Sys., Series B, 6(1) (2006), 69-96.

[29] H. Inaba. Endemic threshold results for age-duration-structured population model for HIV infection. Math. Biosci., 201 (2006), 15-47.

[30] H. Inaba. Age-structured homogeneous epidemic systems with application to the MSEIR epidemic model. J. Math. Biol., 54 (2007), 101-146.

[31] H. Inaba. Homogeneous epidemic systems in the stable population. In: Mathematical Economics RIMS Kokyuroku 1557, Research Institute for Mathematical Sciences, Kyoto University, Kyoto, 2007, 28-44.

[32] A. M. John. Endemic disease in host populations with fully specified demography. Theor. Popul. Biol., 37 (1990), 455-471.

[33] A. M. John. Transmission and control of childhood infectious diseases: Does demography matter? Popul. Stu., 44 (1990), 195-215.

[34] W. Katzmann, K. Dietz. Evaluation of age-specific vaccination strategies. Theor. Popul. Biol., 25 (1984), 125-137.

[35] W. M. Liu, P. van den Driessche. Epidemiological models with varying population size and dose-dependent latent period. Math, Biosci., 128 (1985), 57-69.

[36] P. Manfredi, J. R. Williams. Realistic population dynamics in epidemiological models: the impact of population decline on the dynamics of childhood infectious diseases. Measles in Italy as an example. Math. Biosci., 192 (2004), 153-175.

[37] I. Marek. Iterations of linear bounded operators in non self-adjoint eigenvalue problems and Kellogg's iteration process. Czech. Math. J., 12 (1962), 536-554. 
[38] I. Marek. Frobenius theory of positive operators: Comparison theorems and applications. SIAM J. Appl. Math., 19 (1970), 607-628.

[39] M. Martcheva, H. R. Thieme. Progression age enhanced backward bifurcation in an epidemic model with super-infection. Math. Biol., 46 (2003), 385-424.

[40] R. M. May, R. M. Anderson. Endemic infections in growing populations. Math. Biosci., 77 (1985), 141-156.

[41] A. McLean. Dynamics of childhood infections in high birthrate countries. In: Immunology and Epidemiology, G. W. Hoffmann and T. Hraba (eds.), Lect. Notes Biomath. 65, Springer, Berlin, 1986, 171-197.

[42] A. R. McLean, R. M. Anderson. Measles in developing countries. Part I. Epidemiological parameters and patterns. Epidemiol. Infect., 100 (1988), 111-133.

[43] A. R. McLean, R. M. Anderson. Measles in developing countries. Part II. The predicted impact of mass vaccination. Epidemiol. Infect., 100 (1988), 419-442.

[44] J. A. J. Metz, O. Diekmann. The Dynamics of physiologically structured populations. Lecture Notes in Biomathematics 68, Springer-Verlag, Berlin, 1988.

[45] J. Mossong, C. P. Muller. Estimation of the basic reproduction number of measles during an outbreak in a partially vaccinated population,. Epidemiol. Infect., 124 (2000), 273278.

[46] D. J. Nokes, R. M. Anderson. The use of mathematical models in the epidemiological study of infectious diseases and in the design of mass immunization programmes. Epidemiol. Infect., 101 (1988), 1-20.

[47] A. Pugliese. Population models for diseases with no recovery. J. Math. Biol., 28 (1990), 65-82.

[48] I. Sawashima. On spectral properties of some positive operators. Nat. Sci. Report Ochanomizu Univ., 15 (1964), 53-64.

[49] D. Schenzle. An age-structured model of pre-and post-vaccination measles transmission. IMA J. Math. Appl. Med. Biol. 1 (1984), 169-191.

[50] H. R. Thieme. Epidemic and demographic interaction in the spread of potentially fatal diseases in growing populations. Math. Biosci., 111 (1992), 99-130.

[51] H. R. Thieme. Mathematics in population biology. Princeton University Press, Princeton, 2003.

[52] S. Tuljapurkar, A. M. John. em Disease in changing populations: growth and disequilibrium. Theor. Popul. Biol., 40 (1991), 322-353. 
[53] J. Wallinga, D. Levy-Bruhl, N. J. Gay, C. H. Wachmann. Estimation of measles reproduction ratios and prospects for elimination of measles by vaccination in some Western European countries. Epidemiol. Infect., 127 (2001), 281-295.

[54] J. Wallinga, P. Teunis, M. Kretzschmar. Using data on social contacts to estimate agespecific transmission parameters for respiratory-spread infectious agents. Am. J. Epidemiol., 164 (2006), 936-944.

[55] G. F. Webb. A semigroup proof of the Sharpe-Lotka theorem. In: Infinite-dimensional systems, F. Kappel and W. Schappacher (eds.), Lec. Notes Math., 1076, Springer, Berlin, 1984, 254-268.

[56] G. F. Webb. Theory of nonlinear age-dependent population dynamics. Marcel Dekker, New York, 1985.

[57] H. J. Whitaker, C. P. Farrington. Estimation of infectious disease parameters from serological survey data: the impact of regular epidemics. Stat. Med., 23 (2004), 24292443.

[58] J. R. Williams, P. Manfredi. Ageing populations and childhood infections: the potential impact on epidemic patterns and morbidity. Int. J. Epidemiol., 33 (2004), 566-572. 